\title{
A DEGREE THEORY, FIXED POINT THEOREMS, AND MAPPING THEOREMS FOR MULTIVALUED NONCOMPACT MAPPINGS
}

\author{
BY \\ W. V. PETRYSHYN(1) AND P. M. FITZPATRICK(2)
}

\begin{abstract}
We define and study the properties of a topological degree for ultimately compact, multivalued vector fields defined on the closures of open subsets of certain locally convex topological vector spaces. In addition to compact mappings, the class of ultimately compact mappings includes condensing mappings, generalized condensing mappings, perturbations of compact mappings by certain Lipschitz-type mappings, and others. Using this degree we obtain fixed point theorems and mapping theorems.
\end{abstract}

1. Introduction. The object of this paper is two-fold. First, we develop a degree theory for ultimately compact vector fields defined on closures of open sets lying in certain Hausdorff locally convex topological vector spaces. Second, using this theory, we obtain a number of fixed point theorems and mapping theorems for ultimately compact mappings.

As we shall show in $\S 3$, the class of ultimately compact mappings includes, in addition to multivalued compact mappings, condensing mappings, generalized condensing mappings, and, in particular, perturbations of multivalued compact mappings by certain Lipschitz-type multivalued mappings.

In recent years the Leray-Schauder degree theory for single-valued compact vector fields has been extended, on the one hand, to single-valued mappings of more general type (see Vaĭnikko and Sadovskii [35] for ball-condensing vector fields, Sadovskiî [32] for ultimately compact vector fields, Nussbaum [26] for $k$ set-contractive vector fields, and others (see [33]), and, on the other hand, to multivalued compact vector fields by Granas [13], Cellina and Lasota [4], and Ma [22]).

The degree theory presented in $\$ 2$ extends and unites the degree results of the above authors.

Similarly, the classical fixed point theorems of Banach, Schauder, and Tychonoff have also been extended to more general single-valued mappings (Zabreiko and Krasnosel'skii [36], Browder [2], Kirk [16], Göhde [11], Darbo [6], Sadovskiǐ [32], Nussbaum [26], Furi and Vignoli [9], Petryshyn [29], and others( $\left.{ }^{3}\right)$ ), and to multivalued mappings (Ky Fan [18], Bohnenblust and Karlin [1], Glicksberg [10], Granas [14], Ma [22], Cellina and Lasota [4] for compact mappings; Markin [23], Browder [3] and Reich [31] for multivalued nonexpansive mappings; Himmelberg, Porter and Van Vleck [15] and Danes [5] for multivalued condensing-type

Received by the editors January 15, 1973.

AMS (MOS) subject classifications (1970). Primary 47H10, 47H15.

(1) Supported in part by NSF grant GP-20228.

(2) Supported in part by NSF grant GP-27719.

Copyright $\odot$ 1974, American Mathematical Society 
mappings; and others(3)). Most of the fixed point theorems of the above mentioned authors, in addition to some new fixed point results, will be obtained in $\$ 3$ from the degree theory presented in $\$ 2$.

In \$4 we extend some mapping theorems of $\mathrm{Ma}$ [22] and Granas [13] to multivalued condensing and $k$-set-contractive mappings. In particular, we obtain invariance of domain theorems for the corresponding vector fields.

2. Degree theory for multivalued ultimately compact vector fields. In what follows we will denote by $X$ a separated locally convex topological vector space, which has the additional property that for each compact subset $A$ there is a retraction of $X$ onto $\overline{\text { co }} A$, where by $\overline{\text { co }} A$ we mean the closed convex hull of $A$. If $X$ is metrizable, then, by a theorem of Dugundji [8], $X$ has the latter property (in fact, every closed convex subset is a retract of $X$ ).

If $A \subset X$, we let $K(A)$ and $C K(A)$ denote the families of closed convex and compact convex subsets of $A$, respectively. A multivalued mapping $T$ of $D \subset X \rightarrow 2^{X}$ is called upper semicontinuous (u.s.c.) provided that, whenever $x \in D$ and $V$ is an open set containing $T(x)$, there is an open set $U$ such that $x \in U$ and, if $y \in D \cap U$, then $T(y) \subset V$. If $A \subset D$, then we let $T(A)$ $=\cup_{x \in A} T(x)$, and denote by $\bar{A}$ and $\partial A$ the closure and boundary of $A$, respectively. We recall that a u.s.c. mapping $F: D \rightarrow K(X)$ is called a compact vector field if $(I-F)(D)$ is relatively compact.

In this section we will describe a procedure whereby one may utilize the topological degree defined for multivalued compact vector fields (see Granas [13], $\mathrm{Ma}$ [22], and Cellina and Lasota [4]) in order to define a topological degree for more general classes of mappings: perturbations of the identity by the so-called ultimately compact multivalued mappings. This latter class of mappings includes, in addition to multivalued compact mappings, multivalued contractions, $k$-setcontractions, $k$-ball-contractions, condensing, generalized condensing mappings, and others, which have been recently studied by a number of authors.

To define the class of ultimately compact multivalued mappings we employ a construction utilized by Sadovskii [32] in his development of the index theory for ultimately compact single-valued vector fields.

Let $D \subset X$ be closed and $T: D \rightarrow K(X)$ be u.s.c. We define a transfinite sequence $\left\langle K_{\alpha}\right\rangle$ by induction as follows. Let $K_{0}=\overline{\text { co }} T(D)$. Suppose $\alpha$ is an ordinal such that $K_{\beta}$ has been defined for $\beta<\alpha$. If $\alpha$ is an ordinal of the first kind, let $K_{\alpha}=\overline{\text { co }} T\left(D \cap K_{\alpha-1}\right)$; if $\alpha$ is an ordinal of the second kind, we let $K_{\alpha}=\bigcap_{\beta<\alpha} K_{\beta}$. It is easily verified that the following properties hold for $\left\langle K_{\alpha}\right\rangle$ :

(2.1) each $K_{\alpha}$ is closed and convex with $K_{\alpha} \subseteq K_{\beta}$ for $\alpha \geq \beta$.

(2.2) $T\left(K_{\alpha} \cap D\right) \subset K_{\alpha}$ for each ordinal $\alpha$.

(3) Other contributions have been made in this area (see [3], [33]); we mention only those directly related to our results in this paper. 
Since the transfinite sequence $\left\langle K_{\alpha}\right\rangle$ is nonincreasing, there is an ordinal $\gamma$ such that $K_{\gamma}=K_{\gamma+1}$, and hence $K_{\gamma}=K_{\beta}$ for each $\beta \geq \gamma$. We define $K=K(T, D)$ $=K_{\gamma}$. Then it is clear that $T(D \cap K) \subset K$ and, in fact, that

$$
\overline{\mathrm{co}} T(D \cap K)=K \text {. }
$$

Definition 2.1. A u.s.c. mapping $T: D \rightarrow K(X)$, where $D$ is closed, is called ultimately compact if either $K \cap D=\varnothing$, where $K=K(T, D)$, or if $K \cap D \neq \varnothing$, then $T(D \cap K)$ is relatively compact.

Recalling that $X$ is called quasi-complete if every closed bounded subset of $X$ is complete, we see that when $X$ has this property, then $T: D \rightarrow K(X)$ is ultimately compact if and only if either $K \cap D=\varnothing$ or $K$ is compact. In this case also, our retraction condition on $X$ amounts to the requirement that every convex compact subset of $X$ be a retract of $X$.

Lemma 2.1. Let $D \subset X$ be open with $T: \bar{D} \rightarrow K(X)$ ultimately compact and such that $0 \notin x-T(x)$ for $x \in \partial D$. Assume $K=K(T, \bar{D})$ is such that $K \cap D \neq \varnothing$, and let $\rho$ be a retraction of $X$ onto $K$. Then:

(2.4) if $x \in D$ and $x \in T(x)$, then $x \in K$.

(2.5) $x \in D$ and $x \in T(x)$ if and only if $x \in \overline{\rho^{-1}(D)}$ and $x \in T(\rho(x))$.

Proof. (2.4) Let $x \in D$ and assume that $x \in T(x)$. It is obvious that $x \in K_{0}$. Assume that $\eta$ is an ordinal and $x \in K_{\beta}$ for all $\beta<\eta$. If $\eta$ is an ordinal of the first kind, then since $K_{\eta}=\overline{c o} T\left(K_{\eta-1} \cap \bar{D}\right)$ and $x \in K_{\eta-1} \cap \bar{D}$ we see that $x \in K_{\eta}$. If $\eta$ is an ordinal of the second kind; then since $K_{\eta}=\cap_{\beta<\eta} K_{\beta}$ we see that $x \in K_{\eta}$. Thus it follows that $x \in K_{\gamma}=K$.

(2.5) Assume that $x \in D$ and $x \in T(x)$. Then $x \in K$ by (2.4), and so $\rho(x)=x$, so that $x \in \rho^{-1}(D)$ and $x \in T(\rho(x))$. On the other hand, assume $x \in \overline{\rho^{-1}(D)}$ and $x \in T(\rho(x))$. Since $\rho$ is continuous, $\rho(x) \in \bar{D}$ and, by definition, $\rho(x) \in K$. Thus, by (2.3), $T(\rho(x)) \subset K$, so that $x \in K$ and $x \in T(x)$ with $x \in D$ since $x \notin \partial D$ by assumption.

Definition 2.2. Let $D \subset X$ be open and let $T: \bar{D} \rightarrow K(X)$ be ultimately compact with $0 \notin x-T(x)$ for all $x \in \partial D$. If $K \cap D=\varnothing$, then we define the degree of $I-T$ on $D$ with respect to $0, \operatorname{deg}(I-T, D, 0)$, to be 0 . If $K \cap D \neq \varnothing$, then we let $\rho$ be a retraction of $X$ onto $K$ and define

$$
\operatorname{deg}(I-T, D, 0)=\operatorname{deg}_{c}\left(I-T \rho, \rho^{-1}(D), 0\right),
$$

where the right-hand degree is that defined for compact multivalued vector fields by $\mathrm{Ma}[22]$.

The use of a retraction to define the fixed-point index by means of the LeraySchauder degree appears in Browder (Symposia Math. 2 (1969), Theorem 1). In an unpublished communication, R.D. Nussbaum has shown that one may define a topological degree for single-valued $k$-set-contractive vector fields by means of 
the Leray-Schauder degree together with a retraction onto $K_{\infty}$ (see [25], [26] for the definition of $K_{\infty}$ ). In Definition 2.2 we are using a similar approach.

It follows from (2.5) that the right-hand side of (2.6) is well defined. We now wish to show that the left-hand side of (2.6) is well defined; namely, to show that it is independent of $\rho$. To this end we prove the following

Lemma 2.2. Let $T, D$ and $K$ be as in the previous Definition 2.2. Let $A \subset X$ be the convex closure of a compact set and assume that $A \supset K, A \cap D \neq \varnothing$, $T(A \cap \bar{D}) \subset A$, and $T(A \cap \bar{D})$ is relatively compact. Then, if $\tau$ is a retraction of $X$ onto $A$,

$$
\operatorname{deg}_{c}\left(I-T \tau, \tau^{-1}(D), 0\right)=\operatorname{deg}(I-T, D, 0)
$$

Proof. We must first show that the left-hand side of $(2.7)$ is well defined. Clearly $T\left(\tau\left(\overline{\tau^{-1}(D)}\right)\right)$ is relatively compact. Let $x \in \partial\left(\tau^{-1}(D)\right)$. Then, if $x$ $\in T(\tau(x)), x \in A$, so $x \in \partial D$ and $x \in T(x)$, which is a contradiction.

Now assume that $D \cap K=\varnothing$. Then $T$ has no fixed points, since any fixed point of $T$ must lie in $D \cap K$. We must show that the left-hand side of (2.7) is zero. If this were not the case, then $T \tau$ would have a fixed point in $\overline{\tau^{-1}(D)}$. Such a fixed point would also be a fixed point of $T$ and lie in $D$, which is a contradiction.

We now assume that $D \cap K \neq \varnothing$ and let $\rho$ be any retraction of $X$ onto $K$. Since the fixed point sets of $T \tau$ on $\overline{\tau^{-1}(D)}$ and $T \rho$ on $\overline{\rho^{-1}(D)}$ are both contained in $O=\rho^{-1}(D) \cap \tau^{-1}(D)$, we see, by the additivity of degrees over domains, that it suffices to show that $\operatorname{deg}(I-T \rho, O, 0)=\operatorname{deg}(I-T \tau, O, 0)$.

For $x \in \bar{O}$ and $t \in[0,1]$ define $H_{t}(x)=t T(\rho(x))+(1-t) T(\tau(x))$. Then $H$ is u.s.c. and compact.

We now claim that if $x \in \bar{O}$ and for some $t \in[0,1], x \in H_{t}(x)$, then $x \in T(x)$ and thus $x \notin \partial O$. So assume that $x \in \bar{O}$ and $t \in[0,1]$ are such that $x \in H_{t}(x)$. We note immediately that $x \in A$ and so $\tau(x)=x$, so that $x$ $\in t T(\rho(x))+(1-t) T(x)$, and $x \in \bar{D}$. Since $T(\rho(x))$ and $T(x)$ are in $K_{0}$, and $K_{0}$ is convex, we see that $x \in K_{0}$. Let $\eta$ be an ordinal such that $x \in K_{\beta}$ for $\beta<\eta$. If $\eta$ is an ordinal of the first kind, then $K_{\eta}=\overline{\text { co }} T\left(K_{\eta-1} \cap \bar{D}\right)$. Since $x$ $\in K_{\eta-1} \cap \bar{D}, T(x) \subset K_{\eta}$. But $T(\rho(x)) \subset K$ and since $K \subset K_{\eta}$, with $K_{\eta}$ convex, we see that $x \in K_{\eta}$. Now assume that $\eta$ is an ordinal of the second kind; since $K_{\eta}=\cap_{\beta<\eta} K_{\beta}$ we see that $x \in K_{\eta}$. This shows that $x \in K$. Consequently $\rho(x)=x$ and $x \in T(x)$, since $T(x)$ is convex, and hence $x \in O$. Our conclusion now follows from the invariance under homotopy for multivalued compact vector fields [22]. Q.E.D.

It follows from the above with $A=K$ that our degree is independent of the particular retraction of $X$ onto $K$ chosen.

Before considering the properties of the degree given by Definition 2.2 we will 
show that it is indeed an extension of the degree for compact vector fields defined and studied in [22].

Proposition 2.1. Let $D \subset X$ be open and suppose $T: \bar{D} \rightarrow K(X)$ is compact with $0 \notin x-T(x)$ for each $x \in \partial D$. Then

$$
\operatorname{deg}_{c}(I-T, D, 0)=\operatorname{deg}(I-T, D, 0)
$$

Proof. If $K \cap D=\varnothing$, then the degree on the right is zero by definition. The degree on the left must also be zero, for otherwise $T$ would have a fixed point which would lie in $K \cap D$, so $K \cap D \neq \varnothing$.

If $K \cap D \neq \varnothing$, then since $\overline{T(K \cap D)} \subset \overline{T(\bar{D})}$, we see that $\overline{T(K \cap \bar{D})}$ is compact. Let $\rho$ be a retraction of $X$ onto $K$. Such a retraction exists since $K=\overline{\text { co }} T(\bar{D} \cap K)=\overline{\text { co }} \overline{T(\bar{D} \cap K)}$. Let $O=D \cap \rho^{-1}(D)$. It suffices to show that

$$
\operatorname{deg}_{c}(I-T \rho, O, 0)=\operatorname{deg}_{c}(I-T, O, 0) .
$$

To this end we define for $x \in \bar{O}$ and $t \in[0,1]$

$$
H_{t}(x)=t T(\rho(x))+(1-t) T(x)
$$

and note that $H([0,1] \times \bar{O})$ is relatively compact. That $x \notin H_{t}(x)$ for any $t \in[0,1]$ and $x \in \partial O$ follows from the same argument which has been used in the proof of Lemma 2.2. Hence, by the invariance under homotopy theorem of [22], our conclusion follows. Q.E.D.

Theorem 2.1. Let $T$ and $D$ be as in Definition 2.2. Then if $\operatorname{deg}(I-T, D, 0) \neq 0$, there exists an $x \in D$ with $x \in T(x)$.

Proof. We have $K \cap D \neq \varnothing$. Let $\rho$ be a retraction of $X$ onto $K$. Then, by our hypothesis and Definition 2.2, we see that

$$
\operatorname{deg}_{c}\left(I-T \rho, \rho^{-1}(D), 0\right) \neq 0,
$$

and then there is an $x \in \rho^{-1}(D)$ with $x \in T(\rho(x))$. Thus $x \in T(x)$ by (2.5). Q.E.D.

Theorem 2.2. Let $D \subset X$ be open and let $H:[0,1] \times \bar{D} \rightarrow K(X)$ be u.s.c., and such that $H\left([0,1] \times \bar{D} \cap K^{\prime}\right)$ is relatively compact, where $K^{\prime}=K(H,[0,1] \times \bar{D})$. If $x \notin H_{t}(x)$ for $x \in \partial D$ and $t \in[0,1]$, then

$$
\operatorname{deg}\left(I-H_{0}, D, 0\right)=\operatorname{deg}\left(I-H_{1}, D, 0\right) .
$$

Proof. Let $K_{i}=K\left(H_{i}, \bar{D}\right)$ for $i=0,1$. Then $K_{i} \subset K^{\prime}$, for $i=0$, 1. If $K^{\prime} \cap D$ $=\varnothing$, then $K_{i} \cap D=\varnothing$ for $i=0,1$, and so the conclusion follows. Suppose now that $K^{\prime} \cap D \neq \varnothing$ and note that we have $H_{i}\left(\bar{D} \cap K^{\prime}\right) \subset K^{\prime}$ for $i=0,1$. 
Let $\rho$ be a retraction onto $K^{\prime}$. Then, by Lemma 2.2, it suffices to show that

$$
\operatorname{deg}\left(I-H_{0} \rho, \rho^{-1}(D), 0\right)=\operatorname{deg}\left(I-H_{1} \rho, \rho^{-1}(D), 0\right) .
$$

To this end we define, for $t \in[0,1]$ and $x \in \overline{\rho^{-1}(D)}, F_{t}(x)=H_{t}(\rho(x))$. Then $F$ is u.s.c. and $F\left([0,1] \times \overline{\rho^{-1}(D)}\right) \subset H\left([0,1] \times \bar{D} \cap K^{\prime}\right)$ and is thus relatively compact. Furthermore, if $x \in \overline{\rho^{-1}(D)}$ and $x \in F_{t}(x)$ for some $t \in[0,1]$, then by Lemma 2.1 we see that $x \in D$ and $x \in H_{t}(x)$, so $x \in D$ and therefore $x$ does not lie in $\partial \rho^{-1}(D)$. It follows from the homotopy theorem for multivalued compact vector fields that $(2.8)$ is satisfied. Q.E.D.

Theorem 2.3. Let $T$ and $D$ be as in Definition 2.2. Assume that $D=D_{1} \cup D_{2}$, where $D_{1}$ and $D_{2}$ are open, disjoint, and such that $x \notin T(x)$ if $x \in \partial D_{1} \cup \partial D_{2}$. Then

$$
\operatorname{deg}(I-T, D, 0)=\operatorname{deg}\left(I-T, D_{1}, 0\right)+\operatorname{deg}\left(I-T, D_{2}, 0\right) .
$$

Proof. Let $K=K(T, \bar{D}), K_{1}=K\left(T, \bar{D}_{1}\right)$ and $K_{2}=K\left(T, \bar{D}_{2}\right)$. If $K_{1} \cap D_{1}$ $=K_{2} \cap D_{2}=\varnothing$, then $T$ cannot have any fixed points so that the conclusion follows from Theorem 2.1.

We now consider the case when, say, $K_{1} \cap D_{1} \neq \varnothing$ and $K_{2} \cap D_{2}=\varnothing$. Then the fixed point set of $T$ in $\bar{D}$ is contained in $D_{1}$, and since $K_{1} \subset K$, we have $K \cap D \neq \varnothing$. Let $\rho$ be a retraction of $X$ onto $K$ and let $\tau$ be a retraction of $X$ onto $K_{1}$. Now $K$ plays the role of $A$ in Lemma 2.2 and therefore we have the relation

$$
\operatorname{deg}\left(I-T \rho, \rho^{-1}\left(D_{1}\right), 0\right)=\operatorname{deg}\left(I-T \tau, \tau^{-1}\left(D_{1}\right), 0\right)=\operatorname{deg}\left(I-T, D_{1}, 0\right) .
$$

We now note that the fixed point set of $T \rho$ on $\rho^{-1}(D)$ is contained in $\rho^{-1}\left(D_{1}\right)$, and thus, by [22], since

$$
\operatorname{deg}\left(I-T \rho, \rho^{-1}\left(D_{1}\right), 0\right)=\operatorname{deg}\left(I-T \rho, \rho^{-1}(D), 0\right),
$$

we see that (2.9) is valid since $\operatorname{deg}\left(I-T, D_{2}, 0\right)=0$.

We now consider the case when $K_{1} \cap D_{1} \neq \varnothing$ and $K_{2} \cap D_{2} \neq \varnothing$. Let $\rho$ be a retraction of $X$ onto $K$ and let $\rho_{i}$ be a retraction onto $K_{i}$, for $i=1,2$. It follows from Definition 2.2 and the additivity over domains theorem of [22] that

$$
\begin{aligned}
\operatorname{deg}(I-T, D, 0) & =\operatorname{deg}\left(I-T \rho, \rho^{-1}(D), 0\right) \\
& =\operatorname{deg}\left(I-T \rho, \rho^{-1}\left(D_{1}\right), 0\right)+\operatorname{deg}\left(I-T \rho, \rho^{-1}\left(D_{2}\right), 0\right) \\
& =\operatorname{deg}\left(I-T \rho_{1}, \rho_{1}^{-1}\left(D_{1}\right), 0\right)+\operatorname{deg}\left(I-T \rho_{2}, \rho_{2}^{-1}\left(D_{2}\right), 0\right),
\end{aligned}
$$

the last equality holding since $K$ plays the role of $A$ in Lemma 2.2 for $T: \bar{D}_{1} \rightarrow K(X)$ and for $T: \bar{D}_{2} \rightarrow K(X)$.

We have shown that (2.9) is valid in all cases. Q.E.D.

We now wish to prove a theorem concerning the topological degree of an odd ultimately compact mapping defined on a symmetric neighborhood of the origin. 
To this end we first prove a result for odd multivalued compact vector fields, and for this we will need the following approximation lemma.

Lemma 2.3. Let $D \subset R^{n}$ be compact and let $T: D \rightarrow K\left(R^{n}\right)$ be u.s.c. Assume $D$ is symmetric and $T$ is odd. Then, given $\varepsilon>0$, there exists a single-valued continuous odd mapping $f: D \rightarrow R^{n}$ such that, whenever $x \in D$, there exists $a y \in D$ and $z \in T(y)$ such that $\|x-y\|<\varepsilon$ and $\|f(x)-z\|<\varepsilon$.

Proof. Let $\varepsilon>0$. For each $x \in D$ we may choose $\delta_{x}>0$ such that if $\|x-y\|<2 \delta_{x}$, then $T(y) \subset N_{\varepsilon}(T(x))$. Let $r_{x}=\min \left\{\delta_{x}, \delta_{-x}, \varepsilon\right\}$ for each $x \in D$. Then $\left\{B\left(x, r_{x}\right) \mid x \in D\right\}$ forms an open cover of $D$. Since $D$ is compact we may select $\left\{x_{1}, \ldots, x_{m}\right\} \subset D$ such that $\left\{B\left(x_{i}, r_{x_{i}}\right) \mid i=1, \ldots, m\right\}$ covers $D$; we assume that $\left\{x_{1}, \ldots, x_{m}\right\}=\left\{-x_{1}, \ldots,-x_{m}\right\}$.

Let $\left\{f_{i} \mid i=1, \cdots, m\right\}$ be a partition of unity subordinate to $\left\{B\left(x_{i}, r_{x_{i}}\right) \mid i=1\right.$, $\cdots, m\}$, and for each $i \in\{1, \cdots, m\}$ let $y_{i} \in T\left(x_{i}\right)$. For $x \in D$ define $h(x)=$ $\sum_{i=1}^{m} f_{i}(x) y_{i}$, and then let $f(x)=1 / 2\{h(x)-h(-x)\}$. For convenience let $r_{i}=r_{x_{i}}$.

Let $x \in D$. Choose $k \in\{1, \cdots, m\}$ such that $\left\|x-x_{k}\right\|<r_{k}$ and $r_{i} \leqslant r_{k}$ whenever $\left\|x-x_{i}\right\|<r_{i}$. Then if $\left\|x-x_{i}\right\|<r_{i}$ for some $i \in\{1, \cdots, m\}$, we have $\left\|x_{i}-x_{k}\right\|<$ $2 r_{k}$, so $T\left(x_{i}\right) \subset N_{\epsilon}\left(T\left(x_{k}\right)\right)$, and thus we may choose $z_{i} \in T\left(x_{k}\right)$ with $\left\|z_{i}-y_{i}\right\|<\epsilon$. Let $z=\Sigma_{i=1}^{m} f_{i}(x) z_{i}$; then $z \in T\left(x_{k}\right)$ and $\|h(x)-z\|<\epsilon$.

Now $-x_{k}=x_{l}$ for some $l \in\{1, \cdots, m\}$, and $x_{l}$ plays the same role with respect to $-x$ as $x_{k}$ does with respect to $x$; namely, $\left\|-x-x_{l}\right\|<r_{l}$, and if $\left\|-x-x_{i}\right\|<r_{i}$, then $r_{i} \leqslant r_{l}$. Thus we may select $w \in T\left(x_{l}\right)$ such that $\|h(-x)-w\|<\epsilon$. It follows that $1 / 2\{z-w\} \in T\left(x_{k}\right),\left\|x-x_{k}\right\|<\epsilon$, and $\|f(x)-1 / 2\{z-w\}\|<\epsilon$. Q. E. D.

The previous lemma has been established by Cellina $\left({ }^{4}\right)$ and by C.P. Pixley (in a paper to appear) without the oddness condition on the mapping. We followed a variant of the argument of Pixley.

Before we establish a theorem concerning the degree of an odd ultimately compact mapping, we first obtain, by using Lemma 2.4 and some results of $\mathrm{Ma}$ [22], a corresponding new result for compact vector fields defined on symmetric, but not necessarily convex, neighborhoods of the origin.

Proposition 2.2. Let $O \subset X$ be a symmetric neighborhood of $0 \in X$. Assume that $S: O \rightarrow K(X)$ is an odd multivalued compact mapping such that $x \notin S(x)$ for $x \in \partial O$. Then

$$
\operatorname{deg}_{c}(I-S, O, 0) \text { is odd. }
$$

Proof. We first show that $\{x-S(x)\} \cap \lambda\{-x-S(-x)\}=\varnothing$ for $x \in \partial O$ and $\lambda>0$. If this were not the case, then one could find $x \in \partial O, y \in S(x)$, $w \in S(-x)$, and $\lambda>0$, with $x-y=\lambda(-x-w)$, so

$$
x=\frac{1}{1+\lambda} y+\frac{\lambda}{1+\lambda}(-w)
$$

(4) The authors were unable to find the paper containing his proof. 
But $y,-w \in S(x)$, and thus $x \in S(x)$, which is a contradiction.

Now, by Lemma 9.4 of [22], a result whose proof does not depend on the convexity of $O$, we may find a compact multivalued mapping $F$, whose range lies on a finite dimensional subspace $Y$ of $X$, such that

$$
F(x)=-F(-x) \text { for } x \in \partial(O \cap Y)
$$

and such that

$$
\operatorname{deg}(I-S, O, 0)=\operatorname{deg}\left(\left.(I-F)\right|_{Y}, O \cap Y, 0\right) .
$$

For $x \in \bar{O} \cap Y$ and $t \in[0,1]$, let

$$
H_{t}(x)=(1 /(1+t)) F(x)-(t /(1+t)) F(-x) .
$$

Now, if $x \in \partial(O \cap Y)$, and $x \in H_{t}(x)$, then

$$
x \in(1 /(1+t)) F(x)+(t /(1+t))(-F(-x)) \subset F(x),
$$

which is a contradiction. Thus, since $H([0,1] \times \bar{O} \cap Y)$ has compact closure,

$$
\operatorname{deg}\left((I-F)_{Y}, O \cap Y, 0\right)=\operatorname{deg}\left(I-\left.\frac{1}{2}\{F(x)-F(-x)\}\right|_{Y}, O \cap Y, 0\right) .
$$

For convenience let $T(x)=\frac{1}{2}\{F(x)-F(-x)\}$ for $x \in \bar{O} \cap Y$. Thus $T$ is an odd multivalued mapping with range which has compact closure and $x \notin T(x)$ for $x \in \partial(O \cap Y)$.

Since the fixed point set of $T$ on $\bar{O} \cap Y$ is bounded, we may consider an open, bounded, symmetric neighborhood of 0 , call it $V$, such that the fixed point set is contained in $V$. By the additivity over domain of the topological degree of [22], we see that

$$
\operatorname{deg}(I-T, O \cap Y, 0)=\operatorname{deg}(I-T, O \cap V, 0) .
$$

To prove that this degree is odd, it will suffice to show that there exists a singlevalued odd mapping $f$ defined on $\overline{V \cap} \bar{O}$ such that

$$
x \notin t f(x)+(1-t) T(x) \text { for all } x \in \partial(O \cap V), 0 \leq t \leq 1 .
$$

By Lemma 2.3, for each integer $n$, we may find a single-valued odd mapping $f_{n}: \overline{O \cap V} \rightarrow Y$, which is a $1 / n$-approximation of $T$. We claim that for some $n$ we have

$$
x \notin(1-t) f_{n}(x)+t T(x) \text { for all } x \in \partial(O \cap V), 0 \leq t \leq 1 .
$$

Indeed, if this were not the case, then one could find $\left\{x_{n}\right\} \subset \partial(O \cap V)$, $\left\{t_{n}\right\} \subset[0,1]$, and $w_{n} \in T\left(x_{n}\right)$ for each $n$, with $x_{n}=\left(1-t_{n}\right) f_{n}\left(x_{n}\right)+t_{n} w_{n}$ for each $n$. By compactness, we may assume that $\left\langle t_{n}\right\rangle \rightarrow t_{0},\left\langle w_{n}\right\rangle \rightarrow w_{0}$, and $\left\langle f_{n}\left(x_{n}\right)\right\rangle$ $\rightarrow y_{0}$. Then $\left\langle x_{n}\right\rangle \rightarrow x_{0}$ and, by the u.s.c. and compactness of $T, w_{0} \in T\left(x_{0}\right)$. We now show that $y_{0} \in T\left(x_{0}\right)$. Indeed, let $\varepsilon>0$. Choose $\delta>0$ such that if $\left\|x-x_{0}\right\|<\delta$, then $T(x) \subset N_{e / 2}\left(T\left(x_{0}\right)\right)$. Let $m$ be an integer such that $1 / m$ 
$<\min \{\delta / 2, \varepsilon / 2\}$ and $\left\|x_{n}-x_{0}\right\|<\delta / 2$ if $n \geq m$. Let $n>m$. Then one may choose $y_{n} \in \overline{O \cap V}$ such that $\left\|x_{n}-y_{n}\right\|<1 / n<\delta / 2$, and $z_{n} \in T\left(y_{n}\right)$ with $\left\|z_{n}-f_{n}\left(x_{n}\right)\right\|<1 / n<\varepsilon / 2$. Then $\left\|y_{n}-x_{0}\right\|<\delta$, and thus $z_{n} \in T\left(y_{n}\right)$ $\subset N_{\varepsilon / 2}\left(T\left(x_{0}\right)\right)$. Thus $f_{n}\left(x_{n}\right) \in N_{\varepsilon}\left(T\left(x_{0}\right)\right)$ for all $n \geq m$, and, since $T\left(x_{0}\right)$ is compact, $y_{0} \in T\left(x_{0}\right)$. Consequently $x_{0}=\left(1-t_{0}\right) y_{0}+t_{0} w_{0} \in T\left(x_{0}\right)$ and, since $x_{0} \in \partial(O \cap V)$, we have a contradiction. Q.E.D.

Theorem 2.4. Let $D$ be a symmetric neighborhood of the origin and $T: \bar{D} \rightarrow K(X)$ be an odd ultimately compact mapping such that $x \notin T(x)$ for all $x \in \partial D$. Then $\operatorname{deg}(I-T, D, 0)$ is an odd integer.

Proof. Since $0 \in T(0)$ we see that $K \cap D \neq \varnothing$. Let $\tilde{p}$ be a retraction of $X$ onto $K$. We note that $K$ is a symmetric set, and define $\rho(x)=\frac{1}{2}\{\tilde{p}(x)-\tilde{p}(-x)\}$ for $x \in X$. Then $\rho$ is an odd retraction of $X$ onto $K$. It is easy to see that $\rho^{-1}(D)$ is symmetric. The mapping $T \rho$ is an odd mapping on $\rho^{-1}(D)$. Thus, by Proposition 2.2, $\operatorname{deg}\left(I-T \rho, \rho^{-1}(D), 0\right)$ is an odd integer. Q.E.D.

3. Fixed point theorems for various classes of multivalued ultimately compact mappings. In this section we will utilize the degree theory presented in the previous section to obtain new, as well as some known, fixed point theorems for various classes of multivalued mappings.

It is clear that every compact mapping is ultimately compact. Consequently, the degree theory developed in [4], [13], [22] for multivalued compact vector fields is subsumed by our degree theory for multivalued ultimately compact vector fields. We specifically remark that all of the fixed point theorems proven in this section yield, as special cases, fixed point theorems for multivalued compact mappings.

As a further example of a family of ultimately compact mappings we consider the class of generalized condensing mappings, which has been introduced in [21] in a slightly different fashion for the case of single-valued mappings.

Definition 3.1. Let $T: D \subset X \rightarrow K(X)$ be u.s.c. Then $T$ is called generalized condensing provided that whenever $\Omega \subset D$ is such that $T(\Omega) \subset \Omega$ and $\Omega \mid \bar{\infty} T(\Omega)$ is relatively compact, then $\Omega$ is relatively compact.

The following lemma shows that one may deduce the degree theory and fixed point results for this class of mappings from our previous results. For singlevalued maps similar results were obtained in [32], [21].

Lemma 3.1. Let $D$ be a convex, closed subset of $X$, and let $T: D \rightarrow K(D)$ be generalized condensing. Then $T$ is ultimately compact.

Proof. We know that $K=K(T, D) \subset D$ and, since $\overline{c o} T(K \cap D)=K$, we see that $T(K) \subset K$ and $K \backslash \overline{c o} T(K)=\varnothing$. Thus $K$ is relatively compact, and therefore compact since it is closed. Also, $T(K) \subset K$, so that $T(K)$ is relatively compact. Q.E.D.

Theorem 3.1. Let $D \subset X$ be convex and open and let $T: \bar{D} \rightarrow K(\bar{D})$ be 
ultimately compact with $K=K(T, \bar{D}) \neq \varnothing$. Then $T$ has a fixed point in $\bar{D}$, and if no fixed point of $T$ lies in $\partial D$, then $D \cap K \neq \varnothing$ and $\operatorname{deg}(I-T, D, 0)=1$.

Proof. Assume that $T$ has no fixed points on $\partial D$. Then $D \cap K \neq \varnothing$. Indeed, assume $K \subset \partial D$. Let $V$ be a convex neighborhood of the origin such that $K \subset V$ and let $\rho$ be a retraction of $X$ onto $K$. Consider the mapping $T \rho$, restricted to $\bar{V}$. It is clear that $T(\rho(\bar{V})) \subset K \subset V$, so that $T \rho$ has no fixed points on $\partial V$. The mapping $T \rho{ }_{V}$ is compact and satisfies the Leray-Schauder boundary conditions. It follows from [22] that $T \rho$ has a fixed point in $V$. Thus there exists an $x \in V$ with $x \in T(\rho(x))$. Then $x \in K$, so that $x \in T(x)$. This contradicts the fact that $T$ has no fixed points on $\partial D$. Thus $K \cap D \neq \varnothing$, so we may choose $x_{0} \in K \cap D$.

Let $\rho$ be a retraction of $X$ onto $K$ and for each $x \in \overline{\rho^{-1}(D)}$ and $t \in[0,1]$ let $F_{t}(x)=t T(\rho(x))+(1-t) x_{0}$.

If $x \in \partial \rho^{-1}(D)$ and $0 \leq t \leq 1$ with $x \in t T(\rho(x))+(1-t) x_{0}$, then, since $T(\rho(x)) \subset K$ and $K$ is convex, $x \in K$ and thus $\rho(x)=x$. It follows that $x \in \partial D$ and $x=t z+(1-t) x_{0}$ with $x_{0} \in D$ and $z \in \bar{D}$; this is a contradiction.

It is clear that $F\left([0,1] \times \overline{\rho^{-1}(D)}\right)$ is relatively compact, so that

$$
\operatorname{deg}(I-T, D, 0)=\operatorname{deg}_{c}\left(I-x_{0}, \rho^{-1}(D), 0\right)=1 \text {. Q.E.D. }
$$

Corollary 3.1. Let $D \subset X$ be convex and open. Assume $T: \bar{D} \rightarrow K(\bar{D})$ is ultimately compact, and such that there exists a nonempty subset $A$ of $\bar{D}$ with $\overline{c o} T(A) \supset A$. Then the conclusions of Theorem 3.1 hold.

Proof. In view of Theorem 3.1, it suffices to show that $K=K(T, \bar{D}) \neq \varnothing$. Recalling the construction of $K$, we will show that $A \subset K_{\alpha}$, for each ordinal $\alpha$. Now $K_{0}=\overline{\text { co }} T(\bar{D}) \supset \overline{\text { co }} T(A) \supset A$. Assume that $\eta$ is an ordinal such that $A \subset K_{\beta}$ when $\beta<\eta$. If $\eta$ is of the first kind, then $K_{\eta}=\overline{\text { co }} T\left(K_{\eta-1}\right) \supset \overline{\text { co }} T(A)$ $\supset A$, by the induction hypotheses. If $\eta$ is of the second kind, then since $A \subset K_{\beta}$ for $\beta<\alpha, A \subset \cap_{\beta<\eta} K_{\beta}=K_{\eta}$. Q.E.D.

Corollary 3.2. Let $D$ be an open convex subset of $X$. Assume that $T: \bar{D} \rightarrow K(\bar{D})$ is ultimately compact and that there exists a relatively compact subset $A$ of $D$ with $T(A) \subset A$. Then the conclusions of Theorem 3.1 hold.

Proof. Let $C_{0}=T(\bar{A}) \cap \bar{A}$ and if $C_{n}$ has been defined let $C_{n+1}=T\left(C_{n}\right) \cap \bar{A}$. Then $C_{n+1} \subset C_{n}$ for each $n$, each $C_{n}$ is compact, so $\cap_{n=1}^{\infty} C_{n}=C_{\infty} \neq \varnothing$. The compactness of $\bar{A}$ ensures that $C_{\infty} \subset T\left(C_{\infty}\right)$. Thus $\overline{c o} T\left(C_{\infty}\right) \supset C_{\infty}$, and the result follows from the previous corollary. Q.E.D.

Corollary 3.3. Let $D$ be an open convex subset of $X$, and let $T: \bar{D} \rightarrow K(\bar{D})$ be a generalized condensing mapping. Then the conclusion of Theorem 3.1 holds.

Proof. Let $x_{0} \in \bar{D}$. Define $A=\cup_{n=0}^{\infty} T^{n}\left(x_{0}\right)$, where $T^{0}\left(x_{0}\right)=x_{0}$ and $T^{n+1}\left(x_{0}\right)$ $=T\left(T^{n}\left(x_{0}\right)\right)$. Then $T(A) \subset A$ and $A \backslash \overline{c o} T(A) \subset\left\{x_{0}\right\}$. Thus, by the definition of 
generalized condensing, $A$ is relatively compact. Our result now follows from Corollary 3.2. Q.E.D.

In order to show that our degree is applicable to the study of multivalued contractions, $k$-set-contractions, $k$-ball-contractions, and condensing mappings, which have recently been extensively studied, we first introduce the following notions.

Definition 3.2. Let $C$ be a lattice with a minimal element which we denote by zero, 0 . A mapping $\Phi: 2^{X} \rightarrow C$ is called a measure of noncompactness if for any $D \subset X$ and $B \subset X$ it satisfies the following properties:

(3.1) $\Phi(\overline{c o} D)=\Phi(D)$.

(3.2) $\Phi(D)=0$ if and only if $D$ is precompact.

(3.3) $\Phi(D \cup B)=\max \{\Phi(D), \Phi(B)\}$.

It follows immediately that if $D \subset B$, then $\Phi(D) \leq \Phi(B)$.

In analogy with the single-valued case [32], we now define in terms of $\Phi$ the generalized notion of a condensing mapping.

Definition 3.3. Let $\Phi$ be a measure of noncompactness in $X$ and let $T: D$ $\rightarrow K(X)$ be u.s.c. Then $T$ is said to be $\Phi$-condensing if $\Phi(T(\Omega)) \geq \Phi(\Omega)$ for all $\Omega \subset D$ such that $\Omega$ is not relatively compact. In case $C$ is also linearly ordered, the above condition reduces to the requirement that $\Phi(T(\Omega))<\Phi(\Omega)$ for each $\Omega \subset D$ which is not relatively compact.

We now show that the degree theory developed in $\$ 2$ is applicable to this general class of $\Phi$-condensing mappings.

Lemma 3.2. Let $D \subset X$ be closed and let $T: D \rightarrow K(X)$ be $\Phi$-condensing. If either (a) $D$ is convex and $T(D) \subset D$ or (b) $T: D \rightarrow C K(X)$, then $T$ is ultimately compact.

Proof. Let $K=K(T, D)$. If $K=\varnothing$, there is nothing to prove. Assume that $K \neq \varnothing$. We first consider the case (a): $T(D) \subset D$. Then co $T(K)=K$. Therefore, since $\Phi(K)=\Phi(\overline{c o}(T(K)))=\Phi(T(K)), K$ is relatively compact. Thus $K$, being closed, is compact and, since $\overline{T(K)} \subset K, T(K)$ is relatively compact. have

Now assume $T(x)$ is compact for each $x \in D$. Since co $T(D \cap K)=K$, we

$$
\Phi(T(D \cap K))=\Phi(\overline{c o}(T(D \cap K)))=\Phi(K) \geq \Phi(D \cap K)
$$

and hence $D \cap K$ is relatively compact. Thus $D \cap K$ is compact and, since $T$ is u.s.c. and compact-valued, $T(D \cap K)$ is compact. Q.E.D.

Let us note that every $\Phi$-condensing mapping is generalized condensing and that if $T: D \rightarrow K(X)$ is $\Phi$-condensing then every precompact subset of $D$ is relatively compact.

For later use we need the following

Lemma 3.3. Let $D \subset X$ be open and suppose $H:[0,1] \times \bar{D} \rightarrow K(X)$ is u.s.c. and such that for each $\Omega \subset \bar{D}$, with $\bar{\Omega}$ not compact, we have $\Phi(H([0,1] \times \Omega)) \unlhd \Phi(\Omega)$. Then, if $K=K(H,[0,1] \times \bar{D})$ and $\bar{D} \cap K \neq \varnothing, H([0,1] \times \bar{D} \cap K)$ is compact if 
either (a) $D$ is convex and $H([0,1] \times \bar{D}) \subset \bar{D}$, or (b) $H(t, x) \in C K(X)$ for each $x \in \bar{D}$ and $t \in[0,1]$.

Proof. We know that $\overline{c o} H([0,1] \times \bar{D} \cap K)=K$ and therefore

$$
\Phi(H([0,1] \times \bar{D} \cap K))=\Phi(\overline{c o} H([0,1] \times \bar{D} \cap K))=\Phi(K) \geq \Phi(\bar{D} \cap K)
$$

so that $\bar{D} \cap K$ is relatively compact. Thus $\bar{D} \cap K$ is compact. If (a) holds, then $K=\bar{D} \cap K$ and so $H([0,1] \times \bar{D} \cap K)$ is compact. If (b) holds, then $H$ is a u.s.c., has compact values, and $[0,1] \times \bar{D} \cap K$ is compact; hence, $H([0,1] \times \bar{D} \cap K)$ is compact. Q.E.D.

As we have just shown, if $T: \bar{D} \rightarrow K(X)$ is $\Phi$-condensing and either $T(x)$ is compact for each $x \in D$ or $T(\bar{D}) \subset \bar{D}$, then $T$ is ultimately compact. A trivial example shows that it is not always true that a $\Phi$-condensing mapping is ultimately compact; let $x_{0} \in X$, let $D=\left\{x_{0}\right\}$, and let $T\left(x_{0}\right)=X$. Then $T$ is $\Phi$ condensing with respect to any measure of noncompactness, $K(T, D)=X$, and $T(D \cap K(T, D))=X$.

Theorem 3.2. Let $D \subset X$ be a neighborhood of the origin, and let $T: \bar{D}$ $\rightarrow C K(X)$ be a $\Phi$-condensing mapping such that

$$
\{\lambda x\} \cap T(x)=\varnothing \text { if } x \in \partial D \text { and } \lambda \geq 1 .
$$

Then $\operatorname{deg}(I-T, D, 0)=1$ and thus $T$ has a fixed point in $D$.

Proof. For $x \in \bar{D}$ and $t \in[0,1]$ let $F_{t}(x)=t T(x)$. Let $\Omega \subset \bar{D}$. Suppose that $\Omega$ is not relatively compact and $\Phi(F([0,1] \times \Omega)) \geq \phi(\Omega)$. Then, since $F([0,1]$ $\times \Omega) \subset \overline{c o}(T(\Omega) \cup\{0\}), \quad \Phi(F([0,1] \times \Omega)) \leq \Phi(\overline{c o}(T(\Omega) \cup\{0\}))=\Phi(T(\Omega))$. Hence $\Phi(T(\Omega)) \geq \Phi(\Omega)$ and $\Omega$ is not relatively compact, which is a contradiction. It follows from Lemma 3.3 that $F([0,1] \times \bar{D} \cap K)$ is compact for $K$ $=K(F,[0,1] \times \bar{D})$. Our hypothesis (3.4) clearly implies that $x \notin F_{i}(x)$ for $x \in \partial D$ and $t \in[0,1]$. Thus, by Theorem 2.2, we have

$$
\operatorname{deg}(I-T, D, 0)=\operatorname{deg}(I, D, 0)=1 \text {. Q.E.D. }
$$

Corollary 3.4. Let $D$ be a convex neighborhood of the origin, and assume $T: \bar{D} \rightarrow C K(X)$ is $\Phi$-condensing and such that $T(\partial D) \subset \bar{D}$. Then $T$ has a fixed point.

Proof. If $x \in T(x)$ for some $x \in \partial D$, the result is proven. Assume that $\{\lambda x\} \cap T(x) \neq \varnothing$ for $x \in \partial D$ and $\lambda>1$. Then $\lambda x \in \bar{D}$ and consequently $x \in D$, a contradiction. Thus $T$ satisfies (3.4) and so it has a fixed point. Q.E.D.

Remark 3.1. It follows from Theorem 3.1 and Lemma 3.2 that if in Corollary 3.4 we assume that $T(\bar{D}) \subset \bar{D}$, then one need not require that $T(x)$ be compact for each $x$ in $\bar{D}$.

Remark 3.2. In case $X$ is a Banach space and $T$ is single-valued, Theorem 3.2 was proven in Petryshyn [28] and some of its special cases in [9], [25], [33], while 
for a multivalued compact mapping with $D=\bar{B}(0, r)$ it was proven in Granas [14]. For a multivalued compact mapping acting in a general $X$, the theorem was proven in [22].

In the case that the lattice $C$ in Definition 3.3 has the property that for each $c \in C$ and $\lambda \in R$ with $\lambda>0$ there is defined an element $\lambda c \in C$ with $1 \cdot c=c$, the measure of noncompactness $\Phi: 2^{X} \rightarrow C$ will be called positively homogeneous if for each $\Omega \subset X$ and $\lambda \in R$ with $\lambda \neq 0$ we have $\Phi(\lambda \Omega)=|\lambda| \Phi(\Omega)$.

Theorem 3.3. Let $D \subset X$ be a symmetric neighborhood of the origin. Let $T: \bar{D} \rightarrow C K(X)$ be $\Phi$-condensing with respect to a positively homogeneous measure of noncompactness $\Phi$ and such that

$$
\{x-T(x)\} \cap \lambda\{-x-T(-x)\}=\varnothing \text { for } x \in \partial D \text { and } 0 \leq \lambda \leq 1 .
$$

Then $\operatorname{deg}(I-T, D, 0)$ is an odd integer, so that $T$ has a fixed point.

Proof. For $x \in \bar{D}$ and $t \in[0,1]$, we define

$$
F_{t}(x)=(1 /(1+t)) T(x)-(t /(1+t)) T(-x) .
$$

Suppose $\Omega \subset \bar{D}$ is such that $\Phi(\Omega) \leq \Phi(F([0,1] \times \Omega))$. Now $F([0,1] \times \Omega)$ $\subset \overline{c o}\{T(\Omega) \cup(-T(-\Omega))\}$, so that $\Phi(F([0,1] \times \Omega)) \leq \max \{\Phi(T(\Omega))$, $\Phi(T(-\Omega))\}$. But $\Phi(\Omega)=\Phi(\Omega \cup(-\Omega))$ and $\max \{\Phi(T(\Omega)), \Phi(T(-\Omega))\} \leq \Phi(T(\Omega$ $\cup(-\Omega)))$. Hence we have $\Phi(\Omega \cup(-\Omega)) \leq \Phi(T(\Omega \cup(-\Omega)))$, and so $\Omega \cup(-\Omega)$ is relatively compact, which implies that $\Omega$ is relatively compact. Thus, by Lemma 3.3, $F([0,1] \times K \cap \bar{D})$ is compact, where $K=K(F,[0,1] \times \bar{D})$. We now show that $x \notin F_{t}(x)$ for $x \in \partial D, 0 \leq t \leq 1$. Indeed, if $x \in \partial D$ and $x \in(1 /(1$ $+t)) T(x)-(t /(1+t)) T(-x)$ for some $t \in[0,1]$, then one would have

$$
\frac{1}{1+t} x+\frac{t}{1+t} x \in \frac{1}{1+t} T(x)-\frac{t}{1+t} T(-x)
$$

and so we could choose $z \in T(x)$ and $w \in T(-x)$ with $x-z=t(-x-w)$, which is a contradiction of our hypothesis. From the homotopy invariance theorem and the fact that $\frac{1}{2}\{T(x)-T(-x)\}$ is odd, it follows from Theorem 2.4 that $\operatorname{deg}(I-T, D, 0)$ is an odd integer. Q.E.D.

Remark 3.3. In case $T$ is single-valued the above result appears in [32], [33], while for a compact multivalued map it was proven in [14] for $X$ a Banach space and $D=B(0, r)$ and in [22] for a convex $D$ and general $X$.

We shall now define the class of so-called $k-\Phi$-contractive mappings which, as we shall see later, contains $k$-set-contractions, $k$-ball-contractions, and other mappings as its subclasses, and which have been recently widely studied (see [25], [30], [33]).

Definition 3.4. Let $\Phi: 2^{x} \rightarrow C$ be a measure of noncompactness, where we additionally assume that $C$ is such that for each $c \in C$ and $\lambda \in R$ with $\lambda>0$ there is defined an element $\lambda c \in C$. A u.s.c. mapping $T: D \rightarrow C K(X)$ is called a $k$ - $\Phi$-contraction if $\Phi(T(\Omega)) \leq k \Phi(\Omega)$ for each $\Omega \subset D$ and some $k>0$. 
Lemma 3.4. Let $\Phi: 2^{X} \rightarrow R^{+}=\{t \in R, t \geq 0\} \cup\{\infty\}$ be a measure of noncompactness and suppose that $T: D \rightarrow C K(X)$ is a $k$ - $\Phi$-contraction, $0<k<1$, with $\Phi(T(D)) \in R$. Then $T$ is $\Phi$-condensing if either $X$ is quasi-complete or $D$ is complete.

Proof. Assume that $\Omega \subset D$ is not relatively compact and that $\Phi(T(\Omega))$ $\geq \Phi(\Omega)$. Then, since either $D$ is complete or $X$ is quasi-complete, $\Phi(\Omega) \neq 0$, and since $\Phi(T(D)) \in R$ we have $\Phi(\Omega) \in R$. But $k \Phi(\Omega) \geq \Phi(T(\Omega)) \geq \Phi(\Omega)$ and $0<k<1$. This is a contradiction and hence $T$ is $\Phi$-condensing.

Remark 3.4. In view of Lemma 3.4, Theorems 3.2 and 3.3 are valid for $k-\Phi-$ contractions, $0<k<1$, when $X$ is either quasi-complete or $\bar{D}$ is complete and when $\Phi: 2^{X} \rightarrow R^{+}$is a measure of noncompactness. Note that $\Phi$ is not assumed to be positively homogeneous.

Theorem 3.4. Let $\Phi: 2^{X} \rightarrow R^{+}$be a positively homogeneous measure of noncompactness. Let $D \subset X$ be a neighborhood of the origin and assume that either $\bar{D}$ is

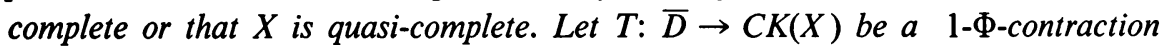
such that $T(\bar{D})$ is bounded in $X$ and $\Phi(T(\bar{D})) \in R$. Furthermore, assume that whenever $\left\{x_{n}\right\} \subset \bar{D}$ and $y_{n} \in T\left(x_{n}\right)$ for each $n$ are such that $x_{n}-y_{n} \rightarrow 0$ as $n \rightarrow \infty$, then there is an $x$ in $\bar{D}$ with $x \in T(x)$. Then, if $T$ satisfies (3.4), Thas a fixed point.

Proof. Let $\left\{\lambda_{n}\right\} \subset(0,1)$ with $\left\langle\lambda_{n}\right\rangle \rightarrow 1$. For each $n$ let $T_{n}=\lambda_{n} T$. Then, since $\Phi$ is positively homogeneous, $T_{n}$ is a $\lambda_{n}-\Phi$-contraction with $\Phi\left(T_{n}(\bar{D})\right) \in R$ and thus $\Phi$-condensing, by Lemma 3.4, with $T_{n}(x)$ compact for each $x \in \bar{D}$. We see that $T_{n}$ satisfies (3.4). Hence, by Theorem 3.2, there exists $x_{n} \in T_{n}\left(x_{n}\right)$ for each $n$. Thus we may select $y_{n} \in T\left(x_{n}\right)$ such that $\left\langle x_{n}-\lambda_{n} y_{n}\right\rangle=0$ and, since $\left\{y_{n}\right\}$ is bounded and $\left\langle\lambda_{n}\right\rangle \rightarrow 1,\left\langle x_{n}-y_{n}\right\rangle=\left\langle\lambda_{n}-1\right\rangle y_{n} \rightarrow 0$ as $n \rightarrow \infty$. Hence, by our condition, there is an $x \in \bar{D}$ such that $x \in T(x)$, i.e., $T$ has a fixed point. Q.E.D.

Remark 3.5. In case $T$ is single-valued, $X$ a Banach space, and $\Phi$ is the set- or ballmeasure of noncompaciness (see the definition below), Theorem 3.4 reduces to the fixed point theorem of Petryshyn [29].

The notion of measure of noncompactness defined by properties (3.1), (3.2), and (3.3) first appeared in Sadovskii [32], who extracted this concept from the setmeasure of noncompactness introduced by Kuratowski [17] and the ball-measure of noncompactness introduced in [12]. The two latter concepts were defined in metric spaces and the set $C$ was the directed set $R^{+}=\{r \in R \mid r \geq 0\} \cup\{\infty\}$, with the usual ordering. We will consider these concepts for separated locally convex spaces.

Assume $\left\{p_{\alpha} \mid \alpha \in A\right\}$ is a family of seminorms which defines the topology on $X$. Given $\alpha \in A$ and $\Omega \subset X$, we define $\chi_{\alpha}(\Omega)=\inf \{\varepsilon>0 \mid$ there exists $\left\{x_{1}, x_{2}, \ldots, x_{n}\right\} \subset X$ with $\left.\Omega \subset \cup_{i=1}^{n}\left\{y \mid p_{\alpha}\left(x_{i}-y\right)<\varepsilon\right\}\right\}$, and $\gamma_{\alpha}(\Omega)=\inf \{d$ $>0 \mid \Omega$ can be contained in the union of a finite number of sets, each of which has $p_{\alpha}$-diameter less that $\varepsilon$. 
We now let $C=\left\{\phi: A \rightarrow R^{+}\right\}$and, if $\phi, \psi \in C$, we define $\phi \leq \psi$ if $\phi(\alpha)$ $\leq \psi(\alpha)$ for all $\alpha \in A$; if $\lambda \in R, \lambda>0$, and $\phi \in C$ we define $\lambda \phi$ by $(\lambda \phi)(\alpha)$ $=\lambda \phi(\alpha)$ for $\alpha \in A$. The zero element of $C$ is defined by $0(\alpha)=0$ for all $\alpha \in A$, and $(\max \{\phi, \psi\})(\alpha)=\max \{\phi(\alpha), \psi(\alpha)\}$ for all $\alpha \in A$. Now define $\chi: 2^{X} \rightarrow C$ by $\chi(\Omega)(\alpha)=\chi_{\alpha}(\Omega)$ and $\gamma: 2^{X} \rightarrow C$ by $\gamma(\Omega)(\alpha)=\gamma_{\alpha}(\Omega)$ for each $\alpha \in A$ and $\Omega \subset X$.

In a locally convex topological vector space the $\chi$-measure of noncompactness was first introduced by Sadovskii [32], while we introduce the $\gamma$-measure of noncompactness here, since the latter is more convenient for studying Lipschitztype maps defined on proper subsets of $X$. Both of these measures are natural extensions of notions studied in Banach and metric spaces in [17], [12], [26], [6], and the proof that they satisfy (3.1), (3.2) and (3.3) follows in the same manner as in the Banach space case. We note that a set $D \subset X$ is bounded in the topological vector space sense if and only if $\gamma_{\alpha}(D)$ or $\chi_{\alpha}(D)$ are finite for each $\alpha \in A$, while $D$ is precompact if and only if $\gamma_{\alpha}(D)=\chi_{\alpha}(D)=0$ for each $\alpha \in A$. Furthermore, $\chi(\lambda \Omega)=|\lambda| \chi(\Omega)$ and $\chi\left(\Omega_{1}+\Omega_{2}\right) \leq \chi\left(\Omega_{1}\right)+\chi\left(\Omega_{2}\right)$ for all $\lambda \in R$ and $\Omega_{1}$ and $\Omega_{2}$ in $X$. Similar properties also hold for the measure $\gamma$.

We note that a mapping $T: D \rightarrow K(X)$ is $\chi$-condensing provided that whenever $\Omega \subset D$, with $\Omega$ not relatively compact, there is an $\alpha \in A$ such that $(\chi(T(\Omega)))(\alpha)$ $<(\chi(\Omega))(\alpha)$. Similarly for $\gamma$-condensing.

Lemma 3.5. Let $T: D \rightarrow C K(X)$ be a $k$ - $\Phi$-contraction, $0<k<1$, where either $\Phi=\chi$ or $\Phi=\gamma$. Then, if $T(D)$ is bounded in the topological vector space sense, $T$ is $\Phi$-condensing provided that either $X$ is quasi-complete or $D$ is complete.

Proof. Let $\Omega \subset D$ with $\Omega$ not relatively compact. Assume that $\Phi(T(\Omega)$ ) $\geq \Phi(\Omega)$. Since $\Omega$ is not relatively compact, and hence not precompact since $D$ is complete or $X$ is quasi-complete, we may choose $\alpha \in A$ with $(\Phi(\Omega))(\alpha)>0$. Now $(\Phi(\Omega))(\alpha) \leq \Phi(T(\Omega))(\alpha)<\infty$, since $T(D)$ is bounded. Furthermore, we have $0<(\Phi(\Omega))(\alpha) \leq \Phi(T(\Omega))(\alpha) \leq k(\Phi(\Omega))(\alpha)<\infty$, and this is clearly a contradiction. Q.E.D.

We remark that when $X$ is metrizable with metric $d$, then we may, as in [17], [12], define a set-measure of noncompactness $\gamma_{d}$ and a ball-measure of noncompactness $\chi_{d}$ which assume values in $R^{+}$and which are known to possess most properties of $\gamma$ and $\chi$ except for the homogeneity property. We add in passing that when $X$ is metrizable, then we see from Lemma 3.4 that if $T: D \rightarrow C K(X)$ is either $\chi_{d}$ - or $\gamma_{d}-k$-contraction and $T(D)$ is bounded in the metric, then $T$ is condensing when $0<k<1$.

In view of Lemmas 3.4 and 3.5, Theorems 3.2 and 3.3 are valid for $k-\Phi$ contractions, $0<k<1$, when either $X$ is quasi-complete or $\bar{D}$ is complete, $T(\bar{D})$ is bounded in the appropriate sense, and $\Phi$ is either $\gamma, \chi, \gamma_{d}$, or $\chi_{d}$. Furthermore, Theorem 3.4 is also valid for the case when $\Phi$ is either $\gamma$ or $\chi$.

We add in passing that besides the set- and ball-measures of noncompactness defined on $2^{X}$, where $X$ is a metric space, one can also define for certain spaces 
other measures of noncompactness whose ranges lie in $R^{+}$. We state two examples (for others see [32]).

If $X=C([a, b])$ we define $\Phi: 2^{X} \rightarrow R^{+}$as follows: $\Phi(\Omega)=+\infty$ if $\Omega \subset X$ is unbounded, while if $\Omega$ is bounded we let

$$
\Phi(\Omega)=\lim _{\delta \rightarrow 0}\left\{\operatorname{Sup}_{f \in \Omega}\{\max \{|f(s)-f(t)||t, s \in[a, b],| s-t \mid<\delta\}\}\right\} .
$$

If $X=L^{p}([a, b])$ we define $\Phi: 2^{X} \rightarrow R^{+}$as follows: $\Phi(\Omega)=+\infty$ if $\Omega$ is unbounded, while if $\Omega$ is bounded we let

$$
\Phi(\Omega)=\lim _{\delta \rightarrow 0}\left\{\operatorname{Sup}_{f \in \Omega}\left\{\operatorname{Sup}_{0 \leq \tau \leq \delta}\left(\int_{a}^{b}|f(t)-f(t+\tau)|^{p} d t\right)^{1 / p}\right\}\right\} .
$$

The former measures of noncompactness have been studied by Sadovskil, Nussbaum, and others.

In what follows we say that $F: X \rightarrow K(X)$ is homogeneous if $F(t x)=t F(x)$ for $x \in X$ and $t \in R$.

We now extend to condensing mappings the result of Lasota and Opial [20] obtained by them for multivalued compact mappings.

Theorem 3.5. Let $X$ be a Banach space and let $G, F: X \rightarrow K(X)$ be condensing with respect to $\Phi$, where $\Phi=\chi_{d}$ or $\Phi=\gamma_{d}$ with $d=\|\|$, on each bounded subset of $X$. Assume also that $F$ is homogeneous and that $x \in F(x)$ only if $x=0$. Suppose that there exists an $\alpha>0$ such that $G(x) \subset F(x)+B(0, \alpha)$ for each $x \in X$. Then $G$ has a fixed point.

Proof. We may choose $\beta>0$ such that $\|x-y\| \geq \beta$ if $\|x\|=1$ and $y$ $\in F(x)$. Indeed, if this were not the case, then one could select $\left\{x_{n}\right\} \subset \partial B(0,1)$ for each $n$ with $\left\|x_{n}-y_{n}\right\|<1 / n$ for some $y_{n} \in F\left(x_{n}\right)$.

Now, $\left\{x_{n}\right\} \subset\left\{y_{n}\right\}+\left\{x_{n}-y_{n}\right\}$ and thus $\Phi\left(\left\{x_{n}\right\}\right) \leq \Phi\left(\left\{y_{n}\right\}\right)+\Phi\left(\left\{x_{n}-y_{n}\right\}\right)$ $\leq \Phi\left(\left\{y_{n}\right\}\right)<\Phi\left(\left\{x_{n}\right\}\right)$, unless $\left\{x_{n}\right\}$ is relatively compact. Thus some subsequence $\left\langle x_{n_{k}}\right\rangle \rightarrow x_{0} \in \partial B$ and $x_{0} \in F\left(x_{0}\right)$, which is a contradiction. We choose $s \in R$ with $s \beta>\alpha$. Then, if $\|x\|=s$ and $y \in F(x)$, we have

$$
\|x-y\|=s\|x / s-y / s\|>s \beta>\alpha,
$$

since $y / s \in F(x / s)$.

Define the homotopy $H:[0,1] \times \bar{B}(0, s) \rightarrow K(X)$ by

$$
H_{t}(x)=F(x)+t(G(x)-F(x))=(1-t) F(x)+t G(x)
$$

for $0 \leq t \leq 1, x \in \bar{B}(0, s)$. Now, if $\Omega \subset \bar{B}(0, s)$, we see that $\Phi(H([0,1] \times \Omega))$ $\leq \Phi(\overline{\mathrm{co}}(F(\Omega) \cup G(\Omega)))<\Phi(\Omega)$, unless $\bar{\Omega}$ is compact. Thus, by Lemma 3.3, $\bar{H}([0,1] \times \bar{D} \cap K)$ is compact, where $K=K(H,[0,1] \times \bar{D})$. Since $\{t(y-z) \mid 0$ $\leq t \leq 1, y \in G(x), z \in F(x)\} \subset B(0, \alpha)$, while $\{x-z \mid x \in \partial B(0, s), z \in F(x)\}$ $\cap \bar{B}(0, \alpha)=\varnothing$, we see that $x \notin H_{t}(x)$ for $x \in \partial B(0, s)$ and $t \in[0,1]$. Thus 
$\operatorname{deg}(I-F, B(0, s), 0)=\operatorname{deg}(I-G, B(0, s), 0)$ and, since $F$ is odd, the left-hand degree is nonzero, so $G$ has a fixed point. Q.E.D.

We now briefly consider fixed point results for ultimately compact mappings defined on sets which need not have nonnull interiors.

Theorem 3.6. Let $D \subset X$ be closed and convex with $T: D \rightarrow K(X)$ ultimately compact and such that $T(D) \subset D$. Then, if $K(T, D) \neq \varnothing, T$ has a fixed point.

Proof. Let $V$ be a convex neighborhood of the origin such that $V \supset K$, and let $\rho$ be a retraction of $X$ onto $K$. Since $K(T \rho, \bar{V}) \supset K(T, D) \neq \varnothing$, it follows from Theorem 3.1 that $T \rho$ has a fixed point in $\bar{V}$, so $T$ has a fixed point in $D$. Q.E.D.

Corollary 3.5. Let $D \subset X$ be closed and convex with $T: D \rightarrow K(X)$ generalized condensing and such that $T(D) \subset D$. Then $T$ has a fixed point.

Proof. $T$ is ultimately compact. By the arguments used in the proofs of Corollaries 3.1, 3.2, and 3.3, we see that $K=K(T, D) \neq \varnothing$. Q.E.D.

Remark 3.6. Since every $\Phi$-condensing mapping is generalized condensing, and, in a quasi-complete space, every $k$ - $\Phi$-contraction, with $0<k<1, \Phi: 2^{X}$ $\rightarrow R^{+}$and with $\Phi(T(D)) \in R$ is $\Phi$-condensing, a result corresponding to the above corollary is valid for these mappings. It is also clear that a result corresponding to Theorem 3.4 for $1-\Phi$-contractions is valid for self-mappings defined on a closed convex set.

Remark 3.7. After the results of the paper were obtained we received a reprint [31] from S. Reich in which Corollary 3.5 has been obtained for $\chi$-condensing mappings satisfying the so-called inward condition, by use of different arguments.

Remark 3.8. Under the additional condition that $T(D)$ is bounded, Corollary 3.5 was established for $\chi$-condensing mappings in [15]. In case $T$ is single-valued, Theorem 3.6 and Corollary 3.5 were first established in Sadovskii [32].

We recall that if $X$ is a metric space with metric $d$, then on $C K(X)$ we may define a metric, the Hausdorff metric, denoted by $d^{*}$, and defined by

$$
d^{*}(A, B)=\max \left\{\sup _{x \in A} \inf _{y \in B} d(x, y), \sup _{x \in B} \inf _{y \in A} d(x, y)\right\} .
$$

We shall call a mapping $T: D \subset X \rightarrow C K(X)$ contractive if $X$ has a metric $d$ and if there exists a $k \in(0,1)$ such that

$$
d^{*}(T(x), T(y)) \leq k d(x, y) \text { for all } x, y \in D ;
$$

the mapping will be called nonexpansive if for each $x, y \in D$ we have

$$
d^{*}(T(x), T(y)) \leq d(x, y) .
$$

Finally, the mapping $T$ will be called completely continuous if whenever $\left\{x_{n}\right\} \subset D$, $\left\langle x_{n}\right\rangle \rightarrow x_{0} \in D$ ( $\rightarrow$ denotes convergence in the weak topology), and $y_{n} \in T\left(x_{n}\right)$ for all $n$, then $\left\langle y_{n}\right\rangle$ has a subsequence which converges strongly to $y_{0} \in T\left(x_{0}\right)$. 
We shall say that the Banach space $X$ satisfies the condition of Opial (see [27]) if whenever $\left\{x_{n}\right\} \subset X$ and $\left\langle x_{n}\right\rangle \rightarrow x_{0}$, then $\lim \inf \left\|x_{n}-x\right\|>\lim \inf \left\|x_{n}-x_{0}\right\|$ for all $x \neq x_{0}$. All uniformly convex Banach spaces with weakly continuous duality mappings have this property; in particular, Hilbert spaces and $l_{p}$ spaces with $p>1$ have this property.

We recall that a mapping $S: D \subset X \rightarrow K(X)$ is called demiclosed if whenever $\left\{x_{n}\right\} \subset D$ and $\left\langle x_{n}\right\rangle \rightarrow x_{0}$ with $y_{n} \in S\left(x_{n}\right)$ for each $n$ such that $\left\langle y_{n}\right\rangle \rightarrow y_{0}$, then $y_{0} \in S\left(x_{0}\right)$.

Lemma 3.6. Let $X$ be a Fréchet space. Let $D \subset X$ be bounded, closed, and convex. Assume that $T: D \rightarrow C K(D)$ is such that there exists a $k \in(0,1)$ with

$$
d^{*}(T(x), T(y)) \leq k d(x, y) \text { for all } x, y \in D .
$$

Then $T$ is ultimately compact and $K=K(T, D) \neq \varnothing$.

Proof. Let $C_{0}=\overline{T(D)}$, and if $C_{n}$ has been defined let $C_{n+1}=\overline{T\left(C_{n}\right)}$. Each $C_{n}$ is nonnull, closed, and $C_{n+1} \subset C_{n} \subset D$ for each $n$. Let $\varepsilon>0$ be such that $0<k^{\prime}=k+\varepsilon<1$.

We claim that if $C_{n}$ can be covered by a finite number of balls of radius $r$, whose centers lie in $C_{n}$, then $C_{n+1}$ can be covered by a finite number of balls of radius $k^{\prime} r$ whose centers lie in $C_{n+1}$. Indeed, assume that $\left\{x_{1}, \ldots, x_{m}\right\} \subset C_{n}$ are such that $C_{n} \subset \cup_{i=1}^{m} B\left(x_{i}, r\right)$. Since each $T\left(x_{i}\right)$ is compact, we may, for each $i$, select $\left\{y_{j}^{i}\right\}_{j=1}^{m(i)} \subset T\left(x_{i}\right)$ and such that $T\left(x_{i}\right) \subset \cup_{j=1}^{m(i)} B\left(y_{j}^{i}, r \varepsilon / 2\right)$. Then $y_{j}^{i}$ $\in C_{n+1}$, for $i \in\{1, \ldots, m\}, j \in\{1, \ldots, m(i)\}$. Now, let $y \in \overline{T\left(C_{n}\right)}=C_{n+1}$. Then one can find $z \in T\left(C_{n}\right)$ with $d(z, y)<r \varepsilon / 2$, and $z \in T(x)$ for some $x \in C_{n}$. Now we can choose $i \in\{1, \ldots, m\}$ with $d\left(x, x_{i}\right)<r$, and thus some $w \in T\left(x_{i}\right)$ with $d(w, z)<k r$. Select $j \in\{1, \ldots, m(i)\}$ such that $d\left(w, y_{j}^{i}\right)<r \varepsilon / 2$. Then it is clear that $d\left(y, y_{j}^{i}\right)<k^{\prime} r$. Thus $C_{n+1} \subset \cup_{i, j} B\left(y_{j}^{i}, k^{\prime} r\right)$, and the claim is justified.

Let $C_{0}$ be covered by a finite number of balls of radius $\beta$. Then, if $r_{n}=\left(k^{\prime}\right)^{n} \beta$, we see that $C_{n}$ can be covered by a finite number of balls of radius $r_{n}$. Now, letting $z_{n} \in C_{n}$ for each $n$, it is clear that we may choose a subsequence of $\left\langle z_{n}\right\rangle$ which converges to some $z_{0}$. Then $z_{0} \in C_{n}$, for each $n$, and so $C_{\infty}=\bigcap_{n=0}^{\infty} C_{n} \neq \varnothing$. It is clear that $T\left(C_{\infty}\right) \subset C_{\infty}$. Moreover, $T\left(C_{\infty}\right)=C_{\infty}$. Indeed, let $x_{0} \in C_{\infty}$. Then for each $n$ one may select $y_{n} \in C_{n}$ and $x_{n} \in T\left(y_{n}\right)$ with $d\left(x_{n}, x_{0}\right)<1 / n$. As before, some subsequence of $\left\langle y_{n}\right\rangle$ converges to some element $y_{0} \in C_{\infty}$ and, by u.s.c. of $T, x_{0} \in T\left(y_{0}\right)$. Thus $T\left(C_{\infty}\right)=C_{\infty}$; since $C_{\infty} \subset K(T, D)$, we have $K(T, D) \neq \varnothing$.

We now show that $K$ is compact, and thus $T$ is ultimately compact. If $K$ is not compact, then $\alpha_{0}=\inf \{\alpha>0 \mid K$ can be covered by a finite number of balls of radius $\alpha$, centered in $K\}>0$. Let $\varepsilon>0$ be such that $k^{\prime}\left(\alpha_{0}+\varepsilon\right)+\varepsilon<\alpha_{0}$, and choose $\left\{x, \ldots, x_{m}\right\} \subset K$, with $K \subset \cup_{i=1}^{m} B\left(x_{i}, \alpha_{0}+\varepsilon\right)$. By the argument used at the beginning of this proof, we may cover $T(K)$ by a finite number of balls of radius $k^{\prime}\left(\alpha_{0}+\varepsilon\right)$, with centers in $T(K) \subset K$. Since $K$ is convex we can cover 
$\overline{c o} T(K)=K$ by a finite number of balls, centered in $K$, of radius $k^{\prime}\left(\alpha_{0}+\varepsilon\right)+\varepsilon$. This contradicts the definition of $\alpha_{0}$. Thus $K$ is compact. Q.E.D.

In view of Lemma 3.6, Theorem 3.6 implies the validity of the following two known fixed point theorems which we mention here as an indication of the generality of Theorem 3.6.

Corollary 3.6. Let $X$ and $D$ be as in Lemma 3.6. If $T: D \rightarrow C K(D)$ is a multivalued contractive mapping, then $T$ has a fixed point in $D$.

Corollary 3.7. Let $X$ be a Banach space which satisfies the condition of Opial. Let $D$ be a convex weakly compact subset of $X$, with $T: D \rightarrow C K(D)$ nonexpansive. Then $T$ has a fixed point.

Proof. Let $z_{0} \in D$ and let $\lambda_{n}=1-1 / n$ for each $n$. Define $T_{n}: D \rightarrow C K(D)$ by $T_{n}(x)=\lambda_{n} T(x)+\left(1-\lambda_{n}\right) z_{0}$. Then $T_{n}$ satisfies the hypothesis of Corollary 3.6 and thus there is an $x_{n} \in D$ with $x_{n} \in T_{n}\left(x_{n}\right)$. Now we may assume $\left\langle x_{n}\right\rangle \rightarrow x_{0}$ $\in D$, and letting $x_{n}=\lambda_{n} y_{n}+\left(1-\lambda_{n}\right) z_{0}$, where $y_{n} \in T\left(x_{n}\right)$, we have $\left\langle x_{n}-y_{n}\right\rangle$ $\rightarrow 0$. But, as has been shown in [19], $I-T$ is demiclosed and thus $T$ has a fixed point. Q.E.D.

Lemma 3.7. Let $X$ be a Fréchet space. Assume $T: X \rightarrow C K(X)$ is such that there is $a k \in(0,1)$ with

$$
d^{*}(T(x), T(y)) \leq k d(x, y) \text { for all } x, y \in X
$$

Then $T$ is $k$-ball-contraction on bounded sets in $X$.

Proof. Let $\Omega \subset X$ be bounded with $\chi(\Omega)=r>0$. Let $\varepsilon>0$. Choose $\left\{x_{1}, \ldots, x_{n}\right\} \subset X$ with $\Omega \subset \cup_{i=1}^{n} B\left(x_{i}, r+\varepsilon\right)$. For each $i \in\{1, \ldots, n\}$ choose $\left\{y_{j}^{i}\right\}_{j=1}^{n(i)}$ with $T(x) \subset \cup_{j=1}^{n(i)} B\left(y_{j}^{i}, \varepsilon\right)$. Then it is clear that

$$
T(\Omega) \subset \bigcup_{i=1}^{n}\left\{\bigcup_{j=1}^{n(i)} B\left(y_{j}^{i}, k(r+\varepsilon)+\varepsilon\right)\right\} .
$$

Thus $X(T(\Omega)) \leq k(r+\varepsilon)+\varepsilon$. Since $\varepsilon$ was arbitrary $\chi(T(\Omega)) \leq k \chi(\Omega)$. Q.E.D.

Remark 3.9. For a multivalued contractive mapping $T$, with contraction constant $k<1$ defined on a proper subset $D$ of $X$, we are unable to show that $T$ is condensing with respect to $\chi$. In fact, such a result has not been proven for single-valued $T$ (it seems to us that the proof of Theorem 2.3 in [32] is in error since there is no guarantee that the centers of the balls defining $\chi(D)$ lie in $D$ ). However, it is not difficult to see that contractions (in fact, the slightly more general type of contractions defined in [32] by means of seminorms on $X$ ) are condensing with respect to $\gamma$ if either $T$ is single-valued or $k<\frac{1}{2}$.

It follows that if $S: X \rightarrow C K(X)$ is contractive and $C: D \rightarrow C K(X)$ is compact, where $D$ is closed and bounded, then $T=S+C: D \rightarrow C K(X)$ is a $k$ ball-contraction providing $X$ is a Banach space and \|\|$=d$. 
Corollary 3.8. Let $X$ be a Banach space with $D$ a bounded (in the metric) neighborhood of $0 \in X$. Let $S: X \rightarrow C K(X)$ be a contraction and $C: \bar{D} \rightarrow C K(X)$ compact. Then, if $T=S+C$ satisfies either (3.4) or (3.5) on $\partial D, T$ has a fixed point.

Proof. From our previous results we see that $T: \bar{D} \rightarrow C K(X)$ is $\chi_{d}$ condensing. The result now follows from Theorems 3.1 and 3.2. Q.E.D.

Remark 3.10. In case $X$ is a Hilbert space and $D=B\left(x_{0}, r\right)$, then Corollary 3.8 remains valid for $S$ only defined on $D$ since, by composing $S$ with the radial retraction, which, by the results of de Figueiredo and Karlowitz [7] is nonexpansive, we see that $S$ can be extended to a contraction on all of $X$. We add in passing that even in the latter case the above corollary appears to be new. In case the contraction constant $k$ satisfies $k<\frac{1}{2}$, then one may show the contraction is a $2 k-\gamma_{d}$ contraction, and thus condensing with respect to $\gamma_{d}$, even if it is defined only on a proper subset of $X$. Using the preceding results and Theorem 3.2 we obtain the following corollary which includes, as special cases, some known fixed point theorems.

Corollary 3.9. Let $X$ be a Banach space which satisfies the condition of Opial, and suppose $D \subset X$ is compact in the weak topology with $0 \in$ int $D$. Assume $C: D$ $\rightarrow C K(X)$ is completely continuous, and $S: X \rightarrow C K(X)$ is nonexpansive. Let $T=S+C: D \rightarrow C K(X)$ and assume $T$ satisfies (3.4) on $\partial D$. Then $T$ has a fixed point.

Proof. For each $n$ let $T_{n}=\lambda_{n} S+\lambda_{n} C$, where $\lambda_{n}=1-1 / n$. Then $T_{n}$ satisfies the conditions of Corollary 3.8 and hence for each $n$ there is some $x_{n} \in D$ with $x_{n} \in T_{n}\left(x_{n}\right)$. Choose $y_{n} \in S\left(x_{n}\right)$ and $z_{n} \in C\left(x_{n}\right)$ with $x_{n}=\lambda_{n}\left(y_{n}+z_{n}\right)$. We may assume that $\left\langle z_{n}\right\rangle \rightarrow z_{0},\left\langle x_{n}\right\rangle \rightarrow x_{0}$, and thus $z_{0} \in C\left(x_{0}\right)$. Then $\left\langle x_{n}-y_{n}\right\rangle \rightarrow z_{0}$ since $\left\langle\lambda_{n}\right\rangle \rightarrow 1$ and, since $I-S$ is demiclosed (see [19]), $z_{0} \in x_{0}-S\left(x_{0}\right)$. Thus $x_{0} \in T\left(x_{0}\right)$. Q.E.D.

Remark 3.11. Corollary 3.6 is valid under weaker assumptions than those presented. Markin [23], and Nadler [24] proved the result without the convexity of $D$ or the assumption that each $T(x)$ is compact and convex. Under the assumption that $X$ is a Hilbert space and $T$ is defined on all of $X$, Corollary 3.7 was proven in Markin [23]. When $X$ is a reflexive Banach space having a strictly convex dual and a weakly continuous duality mapping and $T$ is defined on all of $X$, Corollary 3.7 was obtained by Browder [3]. In its present form Corollary 3.7 was obtained by Lami Dozo [19]. When $X$ is a Banach space with a complete projection scheme and $C$ is single-valued, Corollary 3.9 was proven by Lami Dozo [19]. When $C=0$, Corollary 3.9 was proven by Reich [31].

4. Mapping theorems. This final section is devoted to the derivation of some mapping theorems for condensing mappings and $k-\Phi$-contractions, which, in particular, will include some known results for compact mappings.

To prove an invariance of domain theorem for perturbations of the identity by 
$k$ - $\Phi$-contractions and $\Phi$-condensing mappings we will need the following.

Lemma 4.1. Let $X$ be metrizable with $D \subset X$ open and let $T: \bar{D} \rightarrow C K(X)$ be $\Phi$ condensing with respect to the measure of noncompactness $\Phi$, where $\Phi=\chi$ or $\gamma$. Assume $x \notin T(x)$ for $x \in \partial D$. Then there exists a convex neighborhood of the origin $V$ such that if $S: \bar{D} \rightarrow C K(X)$ is condensing with respect to $\Phi$ and $S(x)$ $C T(x)+V$ for all $x \in \partial D$, then $\operatorname{deg}(I-T, D, 0)=\operatorname{deg}(I-S, D, 0)$.

Proof. We can find a convex neighborhood $V$ of 0 such that $\{x-T(x)\} \cap V$ $=\varnothing$ if $x \in \partial D$. Indeed, if this were not the case, then, letting $\left\{W_{n} \mid n \in N\right\}$ be a monotonically decreasing basis of convex neighborhoods of 0 , one can select, for each $n, y_{n} \in W_{n}, x_{n} \in \partial D$, and $z_{n} \in T\left(x_{n}\right)$ with $y_{n}=x_{n}-z_{n}$. Therefore

$$
\Phi\left(\left\{x_{n}\right\}\right) \leq \Phi\left(\left\{y_{n}\right\}\right)+\Phi\left(\left\{z_{n}\right\}\right)
$$

and, since $\left\langle y_{n}\right\rangle \rightarrow 0, \Phi\left(\left\{y_{n}\right\}\right)=0$. Thus $\Phi\left(\left\{x_{n}\right\}\right) \leq \Phi\left(\left\{z_{n}\right\}\right) \leq \Phi\left(T\left(\left\{x_{n}\right\}\right)\right)$, and it follows that $\left\{x_{n}\right\}$ is relatively compact. So assume $\left\langle x_{n}\right\rangle \rightarrow x_{0}$. Then $x_{0} \in \partial D$ and $x_{0} \in T\left(x_{0}\right)$, which is a contradiction.

Let $S: \bar{D} \rightarrow C K(X)$ be $\Phi$-condensing and such that $S(x) \subset T(x)+V$ for each $x \in \partial D$. Define $H:[0,1] \times \bar{D} \rightarrow C K(X)$ by $H_{t}(x)=T(x)+t(S(x)-T(x))$ $=(1-t) T(x)+t S(x)$. Then, arguing as in Theorem 3.5, we see that, if $K=K(H,[0,1] \times \bar{D})$, then $H([0,1] \times \bar{D} \cap K)$ is compact. If $x \in H_{t}(x)$ for $x \in \partial D$, then $\{x-T(x)\} \cap\{t(S(x)-T(x))\} \neq \varnothing$, so that $\{x-T(x)\} \cap V$ $\neq \varnothing$, which is a contradiction. It follows that

$$
\operatorname{deg}(I-S, D, 0)=\operatorname{deg}(I-T, D, 0) . \quad \text { Q.E.D. }
$$

Theorem 4.1. Assume $X$ is a Banach space. Let $D \subset X$ be open and let $T: D \rightarrow K(X)$ be $k$ - $\Phi$-contractive, $0 \leq k<1$, with respect to a measure of noncompactness $\Phi$, where $\Phi=\chi$ or $\Phi=\gamma$. Assume that for each $x \in D$ there is a convex, symmetric about $x$, neighborhood of $x, V_{x}$, with $V_{x} \subset D$ and such that if $y, \omega \in \bar{V}_{x}$ and $x-\{y-\omega\} \in \partial V$, then $(I-T)(y) \cap(I-T)(\omega)=\varnothing$. Then $(I-T)(D)$ is open.

Proof. Let $y_{0} \in(I-T)(D)$ and choose $x_{0} \in D$ such that $y_{0} \in\left(x_{0}-T\left(x_{0}\right)\right)$. Let $\tilde{T}: V_{x_{0}}-x_{0} \rightarrow K(X)$ be defined by $\tilde{T}(x)=T\left(x+x_{0}\right)+y_{0}-x_{0}$ for $x \in \bar{V}_{x_{0}}$ $-x_{0}$. Then $\tilde{T}: \bar{V}_{x_{0}}-x_{0} \rightarrow K(X)$ is condensing and $0 \in(I-\tilde{T})(0)$. Let $y, w$ $\in \bar{V}_{x_{0}}-x_{0}$ be such that $-\{y-w\} \in \partial\left(\bar{V}_{x_{0}}-x_{0}\right)$. Then $y+x_{0}$ and $w+x_{0}$ and in addition we have

$$
x_{0}-\left\{\left(y+x_{0}\right)-\left(w+x_{0}\right)\right\} \in \partial V_{x_{0}} .
$$

Therefore,

$$
(I-T)\left(y+x_{0}\right) \cap(I-T)\left(w+x_{0}\right)=\varnothing
$$

and hence 


$$
(I-\tilde{T})(y) \cap(I-T)(w)=\varnothing .
$$

From the preceding remarks it is clear that we may assume that $x_{0}=y_{0}=0$.

We will now verify that $\operatorname{deg}\left(I-T, V_{0}, 0\right) \neq 0$. For $x \in \bar{V}_{0}$ and $t \in[0,1]$, we define $H_{t}(x)=T(x /(1+t))-T(-t x /(1+t))$. We know, by a lemma of Nussbaum [26] for single-valued mappings which carries over to multivalued mappings, that for each $\Omega \subset \bar{V}_{0}$ we have

$$
\Phi(H([0,1] \times \Omega)) \leq k \Phi(\Omega),
$$

and hence $H\left([0,1] \times V_{0} \cap K\right)$ is relatively compact, where $K=K(H,[0,1]$ $\left.\times \bar{V}_{0}\right)$. To show that $x \notin H_{t}(x)$ for $x \in \partial V_{0}$ and $t \in[0,1]$, we assume the contrary. Then, for some $x \in \partial V_{0}$ and $t \in[0,1]$, we have

$$
\left\{\frac{1}{1+t} x-T\left(\frac{x}{1+t}\right)\right\} \cap\left\{\frac{-t}{1+t} x-T\left(\frac{-t x}{1+t}\right)\right\} \neq \varnothing
$$

and since $x /(1+t)+t x /(1+t) \in \partial V_{0}$, we obtain a contradiction. Since $H_{1}$ is odd, it follows from Theorems 2.2 and 2.4 that $\operatorname{deg}\left(I-H_{1}, V_{0}, 0\right) \neq 0$. It follows from Theorem 2.1 and Lemma 4.1 that we can find a neighborhood $W$ of the origin with $\operatorname{deg}\left(I-H_{0}-y, V_{0}, 0\right) \neq 0$ for each $y \in W$. Thus $W \subset(I-T)\left(V_{0}\right)$. Q.E.D.

Following Ma [22] we call a mapping $S: D \subset X \rightarrow X$ locally nonopposite if for each $x_{0} \in D$ there is a convex, symmetric neighborhood $V_{0}$ of the origin such that $x_{0}+\bar{V}_{0} \subset D$ and for all $x \in x_{0}+\partial V_{0}$ we have $\lambda\left(x_{0}-x\right) \notin S(x)-S\left(x_{0}\right)$ for $\lambda>0$.

Theorem 4.2. Suppose $X$ is metrizable. Let $D \subset X$ be open and assume $I-T: D \rightarrow C K(X)$ is locally nonopposite with $T \Phi$-condensing, where $\Phi=\gamma$ or $\chi$. Then $(I-T)(D)$ is open.

Proof. Let $z \in(I-T)(D)$ and choose $x_{0} \in D$ with $z \in x_{0}-T\left(x_{0}\right)$. Let $V_{0}$ be a neighborhood corresponding to $x_{0}$ in the definition of locally nonopposite. For $x \in x_{0}+\bar{V}_{0}$ and $t \in[0,1]$ we let $H_{t}(x)=(1-t) x_{0}+t\{T(x)+z\}$. It is clear that $x \notin H_{t}(x)$ for $x \in x_{0}+\partial V_{0}$ because $I-T$ is locally nonopposite. Furthermore, from Lemma 3.3 and Theorem 2.2 we have

$$
\operatorname{deg}\left(I-T-z, x_{0}+V_{0}, 0\right)=\operatorname{deg}\left(I-x_{0}, x_{0}+V_{0}, 0\right)=1 .
$$

Thus, from Lemma 4.1, $z \in \operatorname{int}(I-T)(D)$. Q.E.D.

Corollary 4.1. Assume $X$ is a Banach space and $T: X \rightarrow 2^{X}$ satisfies either the hypothesis of Theorem 4.1 or of Theorem 4.2 with respect to a measure of noncompactness $\Phi$, where $\Phi=\chi$ or $\gamma$. Suppose also that when $\left\langle x_{n}\right\rangle$ is a sequence in $X$ such that $\left\langle x_{n}-y_{n}\right\rangle \rightarrow z$, where $y_{n} \in T\left(x_{n}\right)$ for each $n$, then $\left\{x_{n}\right\}$ is bounded. Then $(I-T)(X)=X$. 
Proof. Since $(I-T)(X)$ is open, it will suffice to show that $(I-T)(X)$ is closed. Indeed, let $\left\langle z_{n}\right\rangle$ be a sequence such that $\left\{z_{n}\right\} \subset(I-T)(X)$ and $\left\langle z_{n}\right\rangle \rightarrow z$. For each $n$, let $z_{n} \in x_{n}-T\left(x_{n}\right)$. Then $\left\langle x_{n}\right\rangle$ is bounded. Now,

$$
\Phi\left(\left\{x_{n}\right\}\right) \leq \Phi\left(\left\{z_{n}\right\}\right)+\Phi\left(\left\{x_{n}-z_{n}\right\}\right) \leq \Phi\left(\left\{T\left(x_{n}\right)\right\}\right),
$$

and therefore $\left\{x_{n}\right\}$ is relatively compact. Assuming $\left\langle x_{n}\right\rangle \rightarrow x$, it follows that $z \in x-T(x)$. Thus $(I-T)(X)$ is closed. Q.E.D.

Remark 4.1. It is easy to see that if in the previous corollary $T(X)$ is assumed to be bounded, then $(I-T)^{-1}(D)$ is bounded when $D$ is precompact.

Remark 4.2. In case $T$ is compact, an examination of our proofs show that Lemma 4.1, Theorems 4.1 and 4.2, and Corollary 4.1 are valid without the assumption that $X$ is metrizable or complete.

Theorem 4.3. Let $X$ be a Banach space, and let $D \subset X$ be open and bounded. Suppose $T: \bar{D} \rightarrow C K(X)$ is $\Phi$-condensing, where $\Phi=\gamma$ or $\Phi=\chi$. Assume also that $\operatorname{deg}(I-T, D, 0) \neq 0$. Then, for each $y \in X, y \neq 0$, there is a $\lambda>0$ such that $\lambda y \in x-T(x)$ for some $x \in \partial D$.

Proof. Assume the conclusion is false. Then there is a $y \in X, y \neq 0$, such that $\{\lambda y \mid \lambda>0\} \cap(I-T)(\partial D)=\varnothing$. Let $\beta>0$, and define $H:[0,1] \times \bar{D}$ $\rightarrow C K(X)$ by $H_{t}(x)=T(x)+t \beta y$. Then $x \notin H_{t}(x)$ for $x \in \partial D$ and $t \in[0,1]$, so that $\operatorname{deg}(I-T-\beta y, D, 0) \neq 0$. Thus, there is some $x \in D$ with $\beta y \in x$ $-T(x)$. Since $\beta>0$ was arbitrary we see that $(I-T)(\bar{D})$ is unbounded, which is a contradiction. Q.E.D.

Corollary 4.2. Assume $X$ is a Banach space. Let $D$ be a bounded, symmetric neighborhood of the origin in $X$, and assume $M$ is a proper subspace of $X$. Then there does not exist an odd $\Phi$-condensing mapping $T: \bar{D} \rightarrow C K(X)$ such that $(I-T)(\bar{D})$ $\subset M \backslash\{0\}$, where $\Phi=\gamma$ or $\Phi=\chi$.

Proof. The result follows immediately from the previous theorem and Theorem 2.4. Q.E.D.

Corollary 4.3. Assume $X$ is a Banach space. Let $D$ be a bounded symmetric neighborhood of the origin in $X$. Assume $M$ is a proper subspace of $X$ with $(I-T)(\bar{D}) \subset M \backslash\{0\}$, where $T$ is $\Phi$-condensing with $\Phi=\gamma$ or $\Phi=\chi$. Then, for some $x \in \partial D,\{x-T(x)\} \cap\{-x-T(-x)\} \neq \varnothing$.

Proof. Assume the conclusion is false. Then, letting $S(x)=\frac{1}{2} T(x)-\frac{1}{2} T(-x)$ for $x \in \bar{D}$, we see that $S$ is $\Phi$-condensing, $S$ is odd, and $(I-S)(\bar{D}) \subset M-\{0\}$. This contradicts the previous corollary. Q.E.D.

Remark 4.3. Theorems 4.1, 4.2 and Corollary 4.1 have been established in [22] for $T$ compact, without the assumption of metrizability on $X$. A result related to Theorem 4.3 has been obtained in [22], where $X$ is assumed to be a general space, $T$ is compact, $D$ is not necessarily bounded, but is convex and symmetric, and the conclusion holds only for $y \in \partial D$. 


\section{REFERENCES}

1. H. F. Bohnenblust and S. Karlin, On a theorem of Ville, Contributions to the Theory of Games, Ann. of Math. Studies, no. 24, Princeton Univ. Press, Princeton, N.J., 1950, pp. 155-160. MR 12, 844.

2. F. E. Browder, Nonexpansive nonlinear operators in a Banach space, Proc. Nat. Acad. Sci. U.S.A. 54 (1965), 1041-1044. MR 32 \#4574.

3. - Nonlinear operators and nonlinear equations of evolution in Banach spaces, Proc. Sympos. Pure Math., vol. 18, Part 2, Amer. Math. Soc., Providence, R.I. (to appear).

4. A. Cellina and A. Lasota, A new approach to the definition of topological degree for multivalued mappings, Atti. Accad. Naz. Lincei Rend. Cl. Sci. Fis. Mat. Natur. (8) 47 (1969), 434-440. MR 43 \#2677.

5. J. Danes, Some fixed point theorems, Comment. Math. Univ. Carolinae 9 (1968), 223-235. MR 38 \#3744.

6. G. Darbo, Punti uniti in transformazioni a condominio non compatto, Rend. Sem. Mat. Univ. Padova 24 (1955), 84-92. MR 16, 1140.

7. D. G. de Figueiredo and L. A. Karlovitz, On the radial projection in normed spaces, Bull. Amer. Math. Soc. 73 (1967), 364-368. MR 35 \#2130.

8. J. Dugundji, An extension of Tietze's theorem, Pacific J. Math. 1 (1951), 353-367. MR 13, 373.

9. M. Furi and A. Vignoli, On a-nonexpansive mappings and fixed points, Atti Accad. Naz. Lincei Rend. Cl. Sci. Mat. Natur. (8) 48 (1970), 195-198. MR 43 \#5513.

10. S. Glicksberg, A further generalization of the Kakutani fixed point theorem with application to Nash equilibrium points, Proc. Amer. Math. Soc. 3 (1952), 170-174. MR 13, 764.

11. D. Göhde, Zum Prinzip der kontraktiven Abbildung, Math. Nachr. 30 (1965), 251-258. MR 32 \#8129.

12. I. C. Gohberg, L. S. Gol'denštein, and A. S. Markus, Investigations of some properties of bounded linear operators with their q-norms, Kišinev, Gos. Univ. Učen. Zap. 29 (1957), 29-36.

13. A. Granas, Sur la notion du degré topologique pour une certaine classe de transformationes multivalentes dans les espaces de Banach, Bull. Acad. Polon. Sci. Sér. Sci. Math. Astronom. Phys. 7 (1959), 191-194. MR 21 \# 7457.

14. - Theorem on antipodes and theorems on fixed points for a certain class of multivalued mappings in Banach spaces, Bull. Acad. Polon. Sci. Sér. Sci. Math. Astronom. Phys. 7 (1959), 271-275. MR 22 \#8365.

15. C. J. Hinmelberg, J. R. Porter and F. S. Van Vleck, Fixed point theorems for condensing multifunctions, Proc. Amer. Math. Soc. 23 (1969), 635-641. MR 39 \#7480.

16. W. A. Kirk, $A$ fixed point theorem for mappings which do not increase distance, Amer. Math. Monthly 72 (1965), 1004-1006. MR 32 \#6436.

17. C. Kuratowski, Sur les espaces complets, Fund. Math. 15 (1930), 301-309.

18. Ky Fan, Fixed point and minimax theorems in locally convex topological linear spaces, Proc. Nat. Acad. Sci. U.S.A. 38 (1952), 121-126. MR 13, 858.

19. E. Lami Dozo, Opérateurs nonexpansifs, P-compact, et propriétés géométriques de la norme, Doctoral Dissertation, Univ. Libre de Bruxelles, 1970.

20. A. Lasota and Z. Opial, Fixed point theorem for multivalued mappings and optimal control problems, Bull. Acad. Polon. Sci. Sér. Sci. Math. Astronom. Phys. 16 (1968), 645-649. MR 40 \# 1832.

21. E. A. Lifšic and B. N. Sadovskii,, $A$ fixed point theorem for generalized condensing operators, Dokl. Akad. Nauk SSSR 183 (1968), 278-279 = Soviet Math. Dokl. 9 (1968), 1370-1371. MR 38 \# 5083.

22. T. W. Ma, Topological degrees for set-valued compact vector fields in locally convex spaces, Dissertationes Math. 92 (1972), 1-43.

23. J. T. Markin, A fixed point theorem for set valued mappings, Bull. Amer. Math. Soc. 74 (1968), 639-640. MR 37 \#3409.

24. S. B. Nadler, Jr., Multivalued contraction mappings, Pacific J. Math. 30 (1969), 475-488. MR 40 \#8035. 
25. R. D. Nussbaum, The fixed point index and fixed point theorems for $k$-set-contractions, Doctoral Dissertation, University of Chicago, Chicago, Ill., 1969.

26. The fixed point index for local condensing maps, Ann. Mat. Pura Appl. 89 (1971), 217-258.

27. Z. Opial, Weak convergence of the sequence of successive approximations for non-expansive mappings, Bull. Amer. Math. Soc. 73 (1967), 591-597. MR 35 \#2183.

28. W. V. Petryshyn, Structure of the fixed point sets of $k$-set-contractions, Arch. Rational Mech. Anal. 40 (1970/71), 312-328. MR 42 \#8358.

29.—, A new fixed point theorem and its applications, Bull. Amer. Math. Soc. 78 (1972), 225-230.

30. - Fixed point theorems for various classes of 1-set-contractive and 1-ball-contractive mappings in Banach spaces, Trans. Amer. Math. Soc. 182 (1973), 323-352.

31. S. Reich, Fixed points in locally convex spaces, Math. Z. 125 (1972), 17-31.

32. B. N. Sadovskii, Measures of noncompactness and condensing operators, Problemy Mat. Anal. Slož. Sistem 2 (1968), 89-119. (Russian)

33.—, Ultimately compact and condensing mappings, Uspehi Mat. Nauk 27 (1972), 81-146. (Russian)

34. H.H. Schaefer, Topological vector spaces, Springer-Verlag, New York, 1970.

35. G. M. Vaĭnikko and B. N. Sadovskiī, On the degree of (ball) condensing vector fields, Problemy Mat. Anal. Slož. Sistem 2 (1968), 84-88. (Russian)

36. P. P. Zabreiko and M. A. Krasnosel'skiī, A method for producing new fixed point theorems, Dokl. Akad. Nauk SSSR 176 (1967), 1233-1235 = Soviet Math. Dokl. 8 (1967), 1297-1299. MR 36 \#3183.

Department of Mathematics, Rutgers University, New Brunswick, New Jersey 08903 (Current address of W. V. Petryshyn)

Courant Institute of Mathematical Sciences, New York University, New York, New YORK 10012

Current address (P. M. Fitzpatrick): Department of Mathematics, University of Chicago, Chicago, Illinois 60637 